\title{
Economic Model Predictive Control of Nonlinear Systems using a Linear Parameter Varying Approach
}

\author{
Fatemeh Karimi-Pour | Vicenç Puig | Carlos Ocampo-Martinez
}

Supervision, Safety and Automatic Control Research Center (CS2AC), Institut de Robòtica i Informàtica Industrial (CSIC-UPC), Universitat Politècnica de Catalunya, Barcelona, Spain

\section{Correspondence}

*Vicenç Puig. Institut de Robòtica i Informàtica Industrial (CSIC-UPC), Universitat Politècnica de Catalunya, Barcelona, Spain. Email: vicenc.puig@upc.edu

\begin{abstract}
Summary
This paper proposes an Economic Model Predictive Control (EMPC) approach for Linear Parameter Varying (LPV) systems. An efficient implementation of the associated MPC optimization problem is introduced based on transforming the LPV model into a linear time-varying one by using an estimation of the scheduling variables along the prediction horizon. This estimation is based on the optimal states/inputs determined from the solution of the previous optimization problem while running the receding horizon strategy. Using this approach, the solution of the proposed LPVbased EMPC scheme would be possible by solving a series of quadratic programming problems at each time instant. This approach allows reducing the computational burden compared to the solution of a nonlinear optimization problem that would result naturally from the LPV-based formulation. The stability of the proposed approach is guaranteed by forcing the terminal state to converge towards the optimal equilibrium/working point of the system. Moreover, the terminal constraint is relaxed by using a constraint set around the terminal state instead of a constraint value and adding a penalty on the terminal state into the cost function. Besides, strict dissipativity is established as a sufficient condition to prove stability. Finally, the effectiveness of the LPV-based EMPC strategy is shown by controlling a small-scale pasteurization system in simulation. The comparison between the proposed control approach and standard MPC approaches is performed. Results show the advantages of the proposed LPV-based EMPC controller in terms of economic cost minimization.
\end{abstract}

\section{KEYWORDS:}

model predictive control, linear parameter varying models, nonlinear systems, economic optimization, stability

\section{1 | INTRODUCTION}

Model predictive control (MPC) has been an active area of research over the last decade and has had significant impact on industrial control engineering because of its ability to deal with multivariable control problems, delays and constraints on system variables and actuators ${ }^{112}$. Moreover, the robustness problem can be addressed regarding model uncertainty and disturbances while still optimizing the established control objectives ${ }^{3}$.

\footnotetext{
${ }^{0}$ Funding Information: This work has been partially funded by the Spanish State Research Agency (AEI) and the European Regional Development Fund (ERFD) through the projects DEOCS (ref. MINECO DPI2016-76493) and SCAV (ref. MINECO DPI2017-88403-R).This work has also been partially funded by AGAUR of Generalitat de Catalunya through the Advanced Control Systems (SAC) group grant (2017 SGR 482).
} 
MPC based on linear time invariant (LTI) models is typically used in process control when systems are operated around a particular set-point since the on-line optimization problem can be formulated as a convex problem by either linear or quadratic programming (QP) approaches. Specifically, in the context of tracking problems addressed through MPC, the cost to be minimized is regularly supposed to be a positive definite function of the state, control input and set-point or reference samples ${ }^{4}$. Nevertheless, for several systems, the nonlinear behavior cannot be neglected. Nonlinear model predictive control (NMPC) is the straightforward way of dealing with systems that involve nonlinear dynamics and constraints. Although efficient nonlinear optimization algorithms have been developed in the last years, optimization problems with nonlinear constraints tend to be computationally more expensive than those related to a linear MPC (LMPC) based on linear/quadratic programming. Moreover, nonlinear programming cannot guarantee that the solution of the nonlinear optimization problem yields the global optimum due to the lack of convexity 5 .

Alternatively, an efficient way of solving the NMPC-related optimization problems is by means of the on-line linearization of the system dynamics at the current state and the control sequences determined during the previous MPC iteration as proposed by the real-time iteration (RTI) approach reported in ${ }^{6}$. In this approach, the nonlinear optimization problem is approximately solved by solving only one properly-formulated QP per sampling time instant. In fact, RTI can be seen as a special case of linear time-varying (LTV) predictive control.

Another way to deal with NMPC is to represent the system behavior by means of (quasi)-linear parameter varying (LPV) models ${ }^{7}$. Using this approach, the on-line linearization is avoided in contrast with the RTI approach. But the LPV model can be transformed into an LTV model by using an estimation of the scheduling variables taking the current state and the control sequence determined in the previous MPC iteration along the prediction horizon as in RTI, leading also to an LTV-based MPCrelated optimization problem able to be efficiently solved through LP/QP approaches. LPV models are a class of linear models whose state-space matrices depend on a set of time-varying parameters ${ }^{8}$. The main advantage of LPV models is that they embed the system nonlinearities in the time-varying parameters, which make the nonlinear system become a linear-like system with time-varying parameters ${ }^{9}$. In this way, LPV models allow applying powerful linear design tools to complex nonlinear models 10.

Conventionally, standard tracking MPC is based on an optimization problem that penalizes the tracking error $\frac{2111}{\text {. Although }}$ this method ensures that the set-point is achieved in a sensible amount of time, it does not guarantee that the transition between set-points is achieved in an economically efficient way. To overcome this problem, MPC has been adapted to solve optimal control problems (OCPs) with general cost functions. In this way, Economic MPC (EMPC) contributes a systematic approach for optimizing an economic performance ${ }^{1213}$.

EMPC has received much attraction because of its capability in integrating real-time process economic optimization and feedback control into an optimal control framework. The optimization problem of EMPC includes three main parts: a cost functional with a stage cost that considers the economic system operational costs to be optimized, system constraints containing state and input constraints as well as other constraints such as stability and operational constraints, and a nonlinear dynamic model to predict the future evolution of the system (and thus, be able to select the optimal input profile with respect to the economic cost over a finite-time prediction horizon) ${ }^{14}$. Unlike tracking MPC, in which target-tracking controllers are calculated by minimizing positive-definite cost functions, EMPC straightly considers general economic functions as stage costs to produce controllers where the stage cost is not necessary positive definite with respect to the optimal steady state, set-point or reference trajectory.

In order to highlight some of the recent theoretical works reported about EMPC, it can be mentioned some studies such as ${ }^{15}$, which forces a terminal constraint into the EMPC formulation to achieve closed-loop stability. Moreover, ${ }^{\sqrt{16}}$ proposes an EMPC controller based on cyclic processes that include closed-loop stability analysis using an appropriate terminal constraint. Lyapunov-based EMPC schemes for nonlinear systems have the ability to manage asynchronous/delayed measurements and allowing a distributed implementation as proposed in 17118 . However, most of the performed research so far has been developed for EMPC and stability conditions based on nonlinear models (see, e.g., ${ }^{1920}$ ) and the extension to the robust case ${ }^{21}$. Moreover, nonlinear EMPC (NEMPC) involves the statement of optimization problems with nonlinear constraints, which may be computationally expensive and, in some cases, there may not guarantee that the solution of the nonlinear optimization problem is the global optimum. Alternatively, a way of solving the optimization problem for a nonlinear system is to transform the nonlinear problem into a QP problem by linearizing the model at each iteration ${ }^{22}$. This method requires updating online the system matrices and the equilibrium/working point when the linearization method is used in case that the operating point changes, which increases the computational burden of the whole control scheme.

The main contribution of this paper is the extension of the (quasi)-LPV approach proposed in ${ }^{\sqrt{23} 24}$ for the design of standard MPC controllers considering nonlinear dynamic models in the case of EMPC. Moreover, an efficient implementation of 
the associated MPC-related optimization problem is proposed based on transforming the LPV model into an LTI one along the prediction horizon using an estimation of the scheduling variables. This estimation is based on the optimal states/inputs determined from the solution of the optimization problem obtained in the previous time instant over the simulation horizon. Using this approach, the solution of the proposed LPV-based EMPC scheme would be possible by solving a series of QP problems at each time instant. This approach allows reducing the computational burden compared to the solution of a nonlinear optimization problem that would result naturally when considering an LPV model. The stability of the proposed approach is guaranteed by pushing the state to the optimum equilibrium point of the system. Moreover, the terminal constraint is relaxed by using a constraint set for the terminal state instead of a point constraint and adding a penalty on the terminal state in the cost function of the related optimization problem. Besides, strict dissipativity is established as a sufficient condition to prove the stability of the whole close-loop scheme. Finally, the effectiveness of the proposed LPV-based EMPC strategy is shown by controlling a small-scale pasteurization system in simulation. The comparison between the LPV-based EMPC and standard MPC approaches is properly discussed. Results show the advantages of the proposed control approach in terms of economic cost minimization.

The remainder of the paper is organized as follows. The problem statement is presented in Section 2 In Section 3 . the formulation of EMPC based on LPV models and iterative prediction scheme are introduced. Then, the stability analysis for EMPC is presented in Section 4 Section 5 describes a pasteurization plant as the case study and states the LPV model and economic control objective of such case study. Then, results of applying the proposed control strategy to the pasteurization system are summarized. Finally, in Section 6, the conclusions of this work are drawn and some research lines for future work are proposed.

\section{Notation}

Throughout this paper, $\mathbb{R}, \mathbb{R}^{n}, \mathbb{R}^{m \times n}, \mathbb{R}_{+}$denote the field of real numbers, the set of column real vectors of length $n$, the set of $m$ by $n$ real matrices and the set of non-negative real numbers, respectively. Similarly, $\rrbracket_{+}$denotes the set of non-negative integer

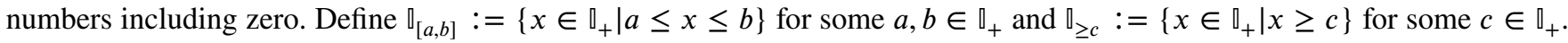
The operator $\oplus$ is direct sum of matrices (block diagonal concatenation). Moreover, $\|$.$\| denotes the spectral norm for matrices.$ The superscript ${ }^{T}$ represents the transpose and operators $<, \leq,=,>, \geq$ denote element-wise relations of vectors. Additionally, $\left[\begin{array}{ll}a & * \\ b & c\end{array}\right]:=\left[\begin{array}{ll}a & b^{\top} \\ b & c\end{array}\right]$

\section{2 | PROBLEM STATEMENT}

\section{1 | LPV representation of the nonlinear system}

Consider a system with nonlinear dynamics represented by the following discrete-time state-space realization:

$$
\begin{aligned}
x(k+1) & =f(x(k), u(k)), \\
y(k) & =g(x(k), u(k)),
\end{aligned}
$$

where the discrete-time variable is denoted as $k \in \mathbb{\mathbb { V }}_{\geq 0}$, the state vector by $x \in \mathbb{R}^{n_{x}}$, the control input vector by $u \in \mathbb{R}^{n_{u}}$, the output vector by $y \in \mathbb{R}^{n_{y}}$, and state-transition maps by $f: \mathbb{R}^{n_{x}} \times \mathbb{R}^{n_{u}} \rightarrow \mathbb{R}^{n_{x}}$ and $g: \mathbb{R}^{n_{x}} \times \mathbb{R}^{n_{u}} \rightarrow \mathbb{R}^{n_{y}}$. The system is subject to state and control constraints

$$
(x(k), u(k)) \in \mathbb{Z}, \quad k \in \mathbb{q}_{\geq 0},
$$

where $\mathbb{Z}:=\left\{(x, u) \in \mathbb{R}^{n_{x} \times n_{u}}, x \in \mathbb{R}^{n_{x}}, u \in \mathbb{R}^{n_{u}} \mid a_{j} x+b_{j} u \leq h_{j}, \forall j \in \mathbb{q}_{\left[1, n_{r}\right]}\right\}$, where $h_{j} \in \mathbb{R}$ is a known bound, $n_{r} \in \mathbb{q}_{\geq 1}$ is the number of constraints and $a_{j} \in \mathbb{R}^{1 \times n_{x}}$ and $b_{j} \in \mathbb{R}^{1 \times n_{u}}, \forall j \in \mathbb{q}_{[1, r]}$. Denote by $\mathbb{X} \subseteq \mathbb{R}^{n_{x}}$ and $\mathbb{U} \subset \mathbb{R}^{n_{u}}$ the projections of $\mathbb{Z}$ on the state and input domains, respectively.

It is assumed that the system has an equilibrium point $\left(x_{s}, u_{s}\right)$ such that $x_{s}=f\left(x_{s}, u_{s}\right)$, and the function model $f(x, u)$ is continuous with respect to the states $x$ and control inputs $u$. The solution of the system for a given sequence of control inputs $\mathbf{u}$ and initial state $x_{0}$ is denoted as $x(k)=\chi\left(k ; x_{0}, u\right), k \in \mathbb{q}_{\geq 0}$.

As discussed in the introduction, instead of directly using the model (1) for designing the EMPC controller, the idea is to transform such a model in a (quasi-)LPV model following the nonlinear embedding approach proposed in ${ }^{9}$ without any linearization process involved. Then, that LPV model is written as

$$
\begin{aligned}
x(k+1) & =A(\theta(k)) x(k)+B(\theta(k)) u(k), \\
y(k) & =C(\theta(k)) x(k)+D(\theta(k)) u(k),
\end{aligned}
$$




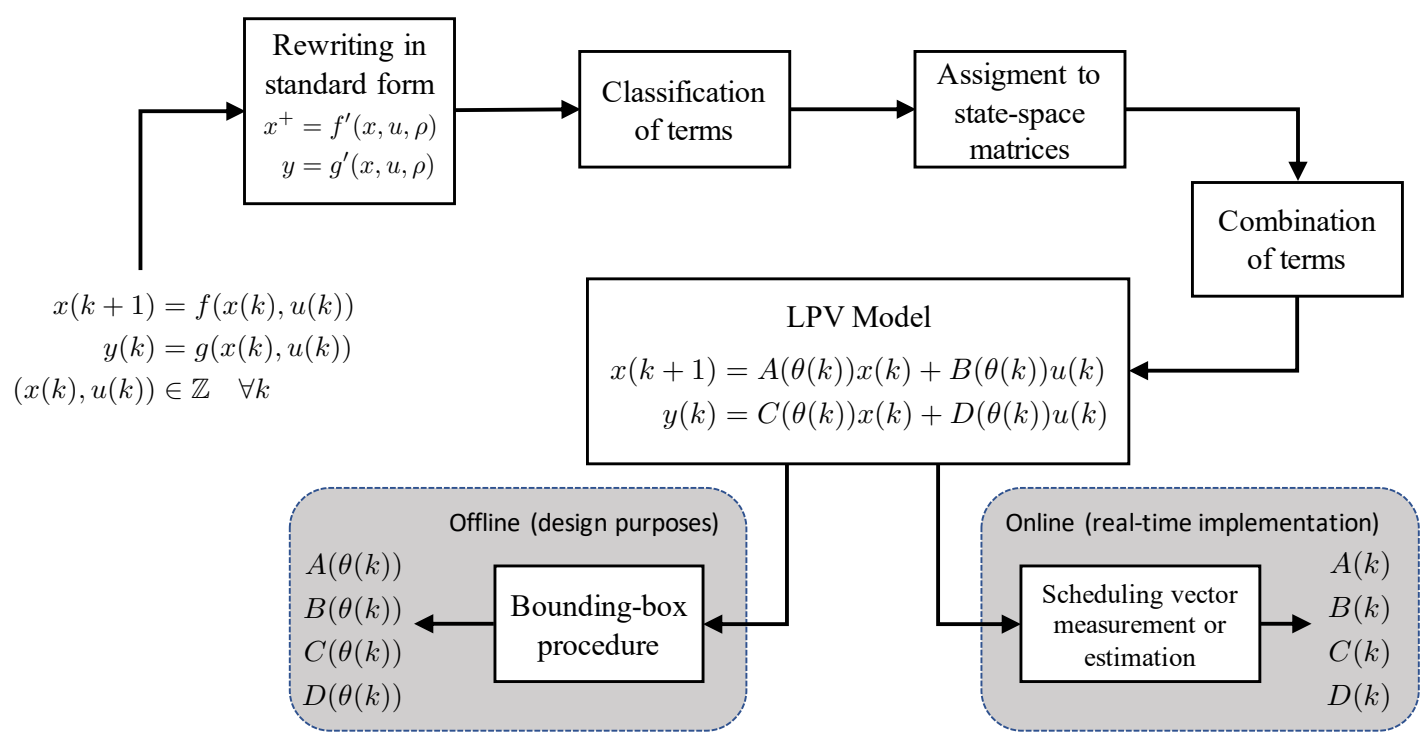

FIGURE 1 Descriptive scheme of the transformation of the system model (1) into the model (3) following the procedure reported in ${ }^{9}$. In this figure and without lost of generality, $x^{+}$denotes the successor step in the evolution of the system state.

where system matrices $A(\theta(k)) \in \mathbb{R}^{n_{x} \times n_{x}}, B(\theta(k)) \in \mathbb{R}^{n_{x} \times n_{u}}, C(\theta(k)) \in \mathbb{R}^{n_{y} \times n_{x}}$ and $D(\theta(k)) \in \mathbb{R}^{n_{y} \times n_{u}}$ are assumed to depend linearly on the parameter vector $\theta(k):=\left[\theta_{1}(k), \theta_{2}(k), \ldots, \theta_{n_{\theta}}\right]^{T} \in \mathbb{R}^{n_{\theta}}$, where $n_{\theta}$ is the number of time-varying parameters. For control design purposes, the LPV model (3) may be used as a bridge between nonlinear system (1) and differential inclusions (DIs) (see ${ }^{\sqrt{25}}$ for more details). The way the system (1) turns into the form in $(3)$ is depicted in Figure 1

In order to derive the proposed EMPC based on LPV systems, Assumptions 1 and 2 are required.

Assumption 1. It is stated that $\theta(k)=\phi(x(k), u(k)) \in \mathbb{R}^{n_{\theta}}$ and $\theta(k) \in \Theta, \quad k \geq 0$, where $\Theta$ is a compact set and $\phi(x, u)$ : $\mathbb{R}^{n_{x}} \times \mathbb{R}^{n_{u}} \rightarrow \mathbb{R}$ is the scheduling function that embeds the nonlinearieties of the time-varying parameters in function of the states and control inputs.

Assumption 2. The pair $(A(\theta), B(\theta))$ is structurally stabilizable for all $\theta \in \Theta$.

In order to prove the stability of the proposed LPV-based EMPC scheme, the LPV model $(3)$ is represented in a polytopic LPV fashion that provides a linear differential inclusion (LDI) of the nonlinear system (1). To do so, consider that the vector $\theta$ belongs to a convex polytope

$$
\Theta:=\left\{\theta(k) \in \mathbb{R}^{n_{\theta}} \mid \theta(k)=\sum_{j=1}^{N} \mu_{j}(\theta(k)) \theta^{j}, \sum_{j=1}^{N} \theta^{j}(k)=1, \mu_{j}(k) \geq 0\right\}
$$

defined by $N$ vertices, where $\theta^{j}$ are the vertices of the polytope and $\mu_{j}(\theta(k))$ are polytopic interpolation functions. Hence, as $\theta(k)$ varies inside the convex polytope $\Theta$, the matrices of the system (3) vary inside a corresponding polytope $\Pi$, which is defined by the convex hull $(\mathrm{Co})$ of $N$ local matrix vertices $\left[A_{j}, B_{j}\right], j \in[1, \ldots, N]$, i.e.,

$$
\Pi:=\operatorname{Co}\left\{\left[\begin{array}{ll}
A_{1} & B_{1}
\end{array}\right],\left[\begin{array}{ll}
A_{2} & B_{2}
\end{array}\right], \cdots,\left[A_{N} B_{N}\right]\right\},
$$

while the system matrices in (3) can be rewritten as

$$
A(\theta(k))=\sum_{j=1}^{N} \mu_{j}(k) A_{j}, \quad B(\theta(k))=\sum_{j=1}^{N} \mu_{j}(k) B_{j} .
$$




\section{2 | EMPC problem formulation}

The considered EMPC-related optimization problem implies the design of a control law that minimizes a specific economic cost index defined here as

$$
L(\tilde{\mathbf{x}}(k), \tilde{\mathbf{u}}(k))=\sum_{i=0}^{H_{p}-1} \ell(x(i), u(i)),
$$

where $\ell(x, u): \mathbb{X} \times \mathbb{U} \rightarrow \mathbb{R}$ represents an economic stage cost, and $\tilde{\mathbf{x}}$ and $\tilde{\mathbf{u}}$ are sequences of states and control inputs along a prediction horizon $H_{p}$, i.e. 1 ,

$$
\begin{aligned}
\tilde{\mathbf{u}}(k) & =\left[\begin{array}{llll}
u(0 \mid k) & \ldots u(1 \mid k) & \ldots & u\left(H_{p}-1 \mid k\right)
\end{array}\right]^{T}, \\
\tilde{\mathbf{x}}(k) & =\left[\begin{array}{llll}
x(1 \mid k) & \ldots x(1 \mid k) & \ldots & x\left(H_{p} \mid k\right)
\end{array}\right]^{T},
\end{aligned}
$$

whose optimal versions are determined by solving the following open-loop OCP at time instant $k$ :

$$
\min _{\tilde{\mathbf{x}}(k), \tilde{\mathbf{u}}(k)} L_{k}(\tilde{\mathbf{x}}(k) \cdot \tilde{\mathbf{u}}(k))
$$

subject to

$$
\begin{aligned}
& x(i+1 \mid k)=f\left(x(i \mid k), u(i \mid k), \quad i \in\left[0, H_{p}-1\right],\right. \\
& u(i \mid k), \in \mathbb{U} \quad i \in\left[0, H_{p}-1\right], \\
& x(i \mid k), \in \mathbb{X} \quad i \in\left[1, H_{p}\right], \\
& x(0 \mid k)=x(k), \\
& x\left(H_{p}\right)=x_{s}^{*} .
\end{aligned}
$$

By solving (8) at every time instant $k$, the economic predictive controller should drive the system towards the optimal reachable steady state, which is determined by using the implicit form of the optimization problem introduced in Definition 1

Definition 1. The optimal reachable steady state and input, $\left(x_{s}^{*}, u_{s}^{*}\right)$, of the system (1) satisfy

$$
\left(x_{s}^{*}, u_{s}^{*}\right)=\arg \min _{(x, u)} \ell(x, u)
$$

subject to

$$
\begin{aligned}
& x=f(x, u), \\
& x \in \mathbb{X}, \\
& u \in \mathbb{U} .
\end{aligned}
$$

In the case of tracking MPC (TMPC), $\ell(x, u)$ is typically designed as a positive definite function with regard to $\left(x_{s}^{*}, u_{s}^{*}\right)$, i.e., $\ell(x, u) \geq 0$ for all $(x, u) \in \mathbb{X} \times \mathbb{U}$ and $\ell(x, u)=0$ if and only if $(x, u)=\left(x_{s}^{*}, u_{s}^{*}\right)$. Hence, the optimal operation often guarantees closed-loop stability of $x_{s}^{*}$ by using the standard MPC stability scheme 26 . On the other hand, in EMPC, $\ell(x, u)$ is formulated taking into account economic criteria such as production costs, energy savings and efficiency, among others. These economic criteria should be either minimized or maximized in terms of profits and environmental issues. Therefore, $\ell(x, u)$ is not necessarily positive definite with regards to $\left(x_{s}^{*}, u_{s}^{*}\right)$. Accordingly, stability properties and convergence in the optimal economic operation are not guaranteed by using the standard MPC stability analysis since these features belong to the positive definiteness of $\ell(x, u)$.

In this paper, the OCP (8) is transformed into the problem presented in Definition 2 by using the LPV representation (3) of the nonlinear system (1).

Definition 2. At each time instant $k$, the following optimization problem should be solved considering that the state $x$ and the vector of parameters $\theta$ are known at the beginning of $H_{p}$ :

$$
\min _{\tilde{\mathbf{x}}(k), \tilde{\mathbf{u}}(k)} L_{k}(\tilde{\mathbf{x}}(k) \cdot \tilde{\mathbf{u}}(k))
$$

\footnotetext{
${ }^{1}$ Here, $m(k+i \mid k)$ denotes the prediction of the variable $m$ at time $k+i$ performed at $k$. For instance, $x(k+i \mid k)$ denotes the prediction of the system state, starting from its initial condition $x(0 \mid k)=x(k)$.
} 
subject to

$$
\begin{aligned}
& x(i+1 \mid k)=A(\theta(i \mid k)) x(i \mid k)+B(\theta(i \mid k)) u(i \mid k), \quad i \in\left[0, H_{p}-1\right], \\
& \theta(i+1 \mid k)=\phi(x(i \mid k), u(i \mid k)), \quad i \in\left[0, H_{p}-1\right], \\
& u(i \mid k), \in \mathbb{U} \quad i \in\left[0, H_{p}-1\right], \\
& x(i \mid k), \in \mathbb{X} \quad i \in\left[0, H_{p}\right], \\
& \theta(0 \mid k)=\theta(k), \\
& x(0 \mid k)=x(k), \\
& x\left(H_{p}\right)=x_{s}^{*},
\end{aligned}
$$

yielding the optimal sequences

$$
\begin{aligned}
\tilde{\mathbf{u}}^{*}(k) & =\left[\begin{array}{llll}
u^{*}(0 \mid k) & \ldots u^{*}(1 \mid k) & \ldots & u^{*}\left(H_{p}-1 \mid k\right)
\end{array}\right]^{T}, \\
\tilde{\mathbf{x}}^{*}(k) & =\left[\begin{array}{llll}
x^{*}(1 \mid k) & \ldots x^{*}(1 \mid k) & \ldots & x^{*}\left(H_{p} \mid k\right)
\end{array}\right]^{T} .
\end{aligned}
$$

The corresponding EMPC law is obtained by applying a receding horizon strategy, i.e., at time instant $k$, only the first element of the optimal control sequence $[10 \mathrm{i})$, namely $u_{\mathrm{EMPC}}(k) \triangleq u^{*}(0 \mid k)$, is applied to the considered system. Then, at time instant $k+1$, a new optimization problem as in (10) is solved.

Remark 1. In case that cost index (7) is not a quadratic function, the resulting optimization problem would not lead to a quadratic optimization problem. To overcome this difficulty, the non-linear function should be transformed into a quadratic form with varying parameters.

Remark 2. Average cost. As discussed in ${ }^{27}$, by the defining the average asymptotic cost of the EMPC law

$$
\bar{L}=\limsup _{H_{p} \rightarrow \infty} \frac{\sum_{i=0}^{H_{p}-1} \ell(x(i), u(i))}{H_{p}},
$$

any feasible trajectory of 10 with terminal constraint $10 \mathrm{~h}$ (i.e., the optimal reachable steady state $x_{s}^{*}$, see Definition 1 will lead to a cost $L$ satisfying

$$
\bar{L} \leq L \text {. }
$$

This result shows that a standard tracking MPC law having as set-point the optimal reachable steady state and input, $\left(x_{s}^{*}, u_{s}^{*}\right)$, will never produce better results at the EMPC law.

\section{3 | PROPOSED LPV-BASED EMPC APPROACH}

Note that the use of (3) into the problem (10) yields in a nonlinear optimization problem. This fact is given since the value of the time-varying parameters $\theta(k)$ along $H_{p}$ depends on the control input and state sequences according to (10c), which are unknown and should be determined by the controller. Indeed, the predicted states depend not only on the future control inputs $u(k), u(k+1), \ldots, u\left(k+H_{p}-1\right)$ (decision variables), but also on the future values of the scheduling parameters $\theta(k)$, which for a pure LPV model are not assumed to be known a priori but only to be measurable online. On the contrary, for a quasi-LPV model, where the scheduling parameters $\theta(k)$ are determined knowing $x(k)$ and $u(k)$ according to Assumption 1, the state trajectory can be predicted using Lemma $11 \mathrm{in}^{28}$, which will be used as the basis for introducing the proposed LPV-based EMPC approach.

Lemma 1. 28. In the quasi-LPV model (10b), the predicted varying parameter vector $\theta(i+1 \mid k)$ in $(10 \mathrm{c})$ can be determined for each $i$ in the prediction horizon $H_{p}$ knowing some estimation of $x(i \mid k)$ and $u(i \mid k)$, fact that states $\hat{\theta}(i \mid k)=\phi(\hat{x}(i \mid k), \hat{u}(i \mid k))$.

For estimating $x(i \mid k)$ and $u(i \mid k)$ along $H_{p}$, the same strategy used into the RTI approach proposed by ${ }^{6}$ will be considered here. That is, since at every MPC iteration the optimal sequences of control inputs and states are quite similar to those obtained at the previous iteration, i.e., the estimated sequences will be obtained from the previous iteration by time-shifting. Thus, instead of solving the optimization problem (10) by using (10b), which will provide the same solution than the EMPC using model (1), 
the proposed solution is to use an approximation based on the $\hat{\theta}$ estimation instead of $\theta$ in $10 \mathrm{~b}]$ This procedure means that the time-varying parameters along $H_{p}$ are estimated and the LPV-based MPC-related optimization problem can be transformed into an LTV-based MPC one. Then, by defining

$$
\Theta(k)=\left[\begin{array}{llll}
\hat{\theta}(0 \mid k) & \hat{\theta}(i+1 \mid k) & \ldots & \hat{\theta}\left(H_{p}-1 \mid k\right)
\end{array}\right]^{T} \in \mathbb{R}^{H_{p} \dot{n}_{\theta}},
$$

which contains the sequence of estimated time-varying parameters along $H_{p}$, the sequence of predicted states in function of the sequence of control inputs (10i) can be expressed as follows:

$$
\tilde{\mathbf{x}}(k)=\mathcal{A}(\Theta(k)) x(k)+\mathcal{B}(\Theta(k)) \tilde{\mathbf{u}}(k),
$$

where

$$
\mathcal{A}(\Theta(k))=\left[\begin{array}{c}
A(\hat{\theta}(k) \\
A(\hat{\theta}(k+1)) A(\hat{\theta}(k)) \\
\vdots \\
A\left(\hat{\theta}\left(k+H_{p}-1\right)\right) A\left(\hat{\theta}\left(k+H_{p}-2\right)\right) \ldots A(\hat{\theta}(k))
\end{array}\right]
$$

and

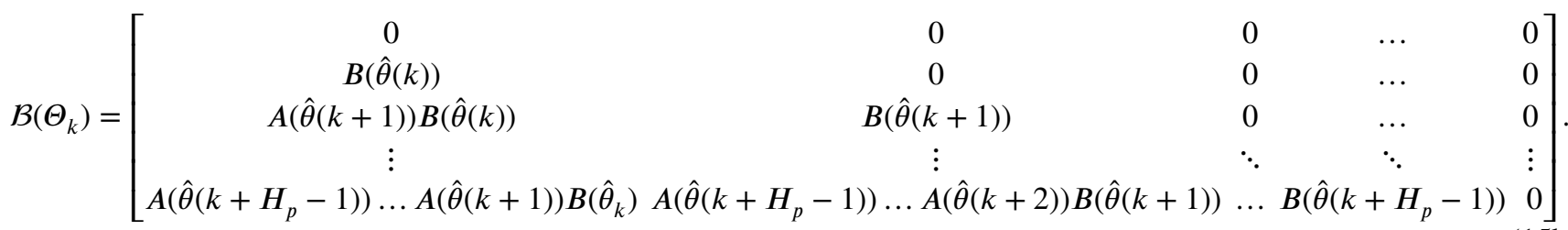

Hence, using (14) the EMPC optimization problem (10) can be rewritten as

$$
\min _{\tilde{\mathbf{u}}(k)} L_{k}(\mathcal{A}(\Theta(k)) x(k)+\mathcal{B}(\Theta(k)), \tilde{\mathbf{u}}(k))
$$

subject to

$$
\begin{array}{ll}
u(i \mid k) \in \mathbb{U}, & i \in\left[0, H_{p}-1\right], \\
x(i \mid k) \in \mathbb{X}, & i \in\left[0, H_{p}\right], \\
\theta(0 \mid k)=\theta(k), & \\
x(0 \mid k)=x(k), & \\
x\left(H_{p}\right) \in \mathbb{X}_{f} . &
\end{array}
$$

where the calculation of the terminal set $\mathbb{X}_{\mathbb{f}}$ will be presented later in the paper.

In this way, the online EMPC-related optimization problem can be solved as a QP problem, which is significantly more efficient than solving a nonlinear optimization problem. The parameter varying estimation will be done by means of the following approach at each discrete time $k$ :

- In the first iteration $(k=0)$, the optimization problem $(10)$ is solved as a linear problem since the LPV model $(10 \mathrm{c})$ is replaced by the LTI model that is obtained considering $\theta(0 \mid k)=\theta(1 \mid k)=\theta(2 \mid k)=\ldots=\theta\left(H_{p}-1 \mid i\right)$ along $H_{p}$ and where $\theta(0 \mid k)=\phi(x(0), u(0))$ and $u(0)=0$.

- In the sequel and according to Lemma 1 , the sequence of the estimated time-varying parameters $\Theta(k)$ in $(13)$ is obtained by using $\hat{\theta}(i \mid k)=\phi(\hat{x}(i \mid k), \hat{u}(i \mid k))$, where the estimated states and control inputs are obtained from the optimal state and control input sequences, $\tilde{\mathbf{x}}^{*}(k-1)$ and $\tilde{\mathbf{u}}^{*}(k-1)$, obtained from solving $(16)$ in the previous iteration and conveniently shifting them in time. 


\section{4 | STABILITY AND CONVERGENCE ANALYSES}

In 27, conditions for feasibility, performance and stability of EMPC for nonlinear systems are revisited. Here, the particular conditions for the LPV-based EMPC approach will be derived.

\section{1 | Stability analysis}

In Section 3. the proposed EMPC approach is provided by considering the terminal equality constraint $x\left(H_{p}\right)=x_{s}^{*}$. However, this constraint cannot guarantee the stability of EMPC based on the LPV models since that constraint is defined just around a single point. In this section, it will be shown that, by considering the terminal state belongs to a compact set, the stability of the closed-loop scheme can be ensured.

By following 304 , stability of NEMPC schemes requires imposing a constraint region on the terminal state instead of a point constraint (16f) and adding a penalty on the terminal state in the cost function (7). Hence, the economic objective function is defined as

$$
L(\tilde{\mathbf{x}}(k), \tilde{\mathbf{u}}(k)):=\sum_{i=0}^{H_{p}-1} \ell(x(i), u(i))+V_{f}\left(x\left(H_{p}\right)\right),
$$

where $V_{f}: \mathbb{X}_{f} \rightarrow \mathbb{R}$ is the penalty on the terminal state set, and $\mathbb{X}_{f} \subseteq \mathbb{X}$ is a compressed terminal region including the steady-

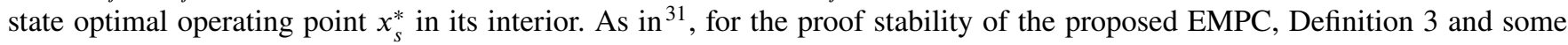
assumptions are next introduced.

Definition 3. System (3) is dissipative with respect to the stock rate $s: \mathbb{X} \times \mathbb{U} \rightarrow \mathbb{R}$, whereas there exists a function $\gamma: \mathbb{X} \rightarrow \mathbb{R}$

$$
\gamma(A(\theta(k)) x(k)+B(\theta(k)) u(k))-\gamma(x) \leq s(x, u),
$$

for all $(x, u) \in \mathbb{Z} \subseteq \mathbb{X} \times \mathbb{U}$. Additionally, if $\rho: \mathbb{X} \rightarrow \mathbb{R}_{\geq 0}$ is definite positive ${ }^{3}$ then

$$
\gamma\left(A\left(\theta_{k}\right) x(k)+B(\theta(k)) u(k)\right)-\gamma(x) \leq-\rho\left(x-x_{s}^{*}\right)+s(x, u),
$$

and it can be said the system is strictly dissipative.

Assumption 3. (Strict dissipativity) System (3) is strictly dissipative with respect to the stock rate $s(x, u):=\ell(x, u)-\ell\left(x_{s}^{*}, u_{s}^{*}\right)$.

Assumption 4. The stage cost $\ell$ and model (3) are continuous on $\mathbb{Z}$. The terminal cost function $V_{f}($.$) is continuous in \mathbb{X}_{f}$.

Assumption 5. The storage function $\gamma(k)$ is continuous in $\mathbb{Z}$.

Assumption 6. (Stability assumption) Given a compact terminal region $\mathbb{X}_{f} \subseteq \mathbb{X}$, containing the point $x_{s}^{*}$ in its interior, there exists a control law $K_{f}(\theta(k)): \mathbb{X}_{f} \rightarrow \mathbb{U}$ such that the following condition holds:

$$
V_{f}\left(\left(A(\theta(k))+B(\theta(k)) K_{f}(\theta(k))\right) x(k)\right) \leq V_{f}(x)-\ell\left(x, K_{f}(\theta(k)) x(k)\right)+\ell\left(x_{s}^{*}, u_{s}^{*}\right) .
$$

Remark 3. Assumption 6implies that the set $\mathbb{X}_{f}$ for all $k \in \mathbb{1}_{\geq 0}$ is invariant under the control law defined by $u(k)=K_{f}(\theta(k)) x(k)$. $\diamond$

Remark 4. Considering Assumption 6 is the only condition on $V_{f}$, it can be assumed without loss of generality that $V_{f}\left(x_{s}^{*}\right)=0$ for all $k \in \mathbb{\mathbb { l }}_{\geq 0}$. It should be remarked that unlike the standard MPC problem, $V_{f}(x)$ is not necessarily positive definite with respect to $x_{s}^{*}$.

In order to analyse the asymptotic stability of the EMPC-based closed-loop system, the following rotated cost function with terminal costs is considered:

$$
\begin{aligned}
\mathcal{L}(x, u) & :=\ell(x, u)-\ell\left(x_{s}^{*}, u_{s}^{*}\right)+\gamma(x)-\gamma(A(\theta(k)) x(k)+B(\theta(k)) u(k)), \\
\bar{V}_{f}(x) & :=V_{f}(x)-V_{f}\left(x_{s}\right)+\gamma(x)-\gamma\left(x_{s}\right) .
\end{aligned}
$$

\footnotetext{
${ }^{3} \mathrm{~A}$ function is positive definite according to some points $x_{s}^{*} \in \mathbb{X}$ if it is of continuous nature, $\rho\left(x_{s}^{*}\right)=0$ and $\rho(x) \geq 0$ for all $x \neq x_{s}^{*}$.
} 
With the costs $\bar{V}_{f}$ and $\mathcal{L}$, the following auxiliary OCP is introduced:

$$
\min _{\tilde{\mathbf{x}}(k), \tilde{\mathbf{u}}(k)} \bar{L}(\tilde{\mathbf{x}}(k), \tilde{\mathbf{u}}(k)):=\sum_{i=0}^{H_{p}-1} \mathcal{L}(x(i), u(i))+\bar{V}_{f}\left(x\left(H_{p}\right)\right),
$$

subject to (16b)-(16f).

Since constraints in problem (22) are the same as in problem [16, both problems have an identical feasible set for all $k \in \mathbb{1}_{\geq 0}$. And, consequently, solutions exist for both problems for $x \in \mathbb{X}\left(H_{p)}\right.$.

Remark 5. According to Assumption 6 it can be assumed that $\gamma\left(x_{s}^{*}\right)=0$ for all $k \in \mathbb{1}_{\geq 0}$ without loss of generality.

Lemma 2. Consider Assumptions 45 and 6hold. The solution of the auxiliary problem (22) is identical to the solution of the original problem 16 .

Proof. By considering that both problems only differ in the cost function, expanding the rotated cost function yields

$$
\begin{aligned}
\bar{L}(\tilde{\mathbf{x}}(k), \tilde{\mathbf{u}}(k)) & =\sum_{i=0}^{H_{p}-1} \mathcal{L}(x(i), u(i))+\bar{V}_{f}\left(x_{H_{p}}\right) \\
& =\sum_{i=0}^{H_{p}-1} \ell(x(i), u(i))-\ell\left(x_{s}^{*}, u_{s}^{*}\right)+\gamma(x)-\gamma\left(A(\theta(i)) x_{i}+B(\theta(i)) u(i)\right)+V_{f}\left(x_{H_{p}}\right)-V_{f}\left(x_{s}^{*}\right)+\gamma\left(x_{H_{p}}\right)-\gamma\left(x_{s}^{*}\right) \\
& \left.=L(\tilde{\mathbf{x}}(k), \tilde{\mathbf{u}}(k))-V_{f}\left(x_{s}^{*}\right)+\gamma\left(x_{H_{p}}\right)\right)-\gamma\left(x_{s}^{*}\right)+\gamma(x)-\gamma\left(x_{H_{p}}\right)-\sum_{i=0}^{H_{p}-1} \ell\left(x_{s}^{*}, u_{s}^{*}\right) .
\end{aligned}
$$

Subsequently, according to Remark 3, it is obtained that

$$
\bar{L}(\tilde{\mathbf{x}}(k), \tilde{\mathbf{u}}(k))=L(\tilde{\mathbf{x}}(k), \tilde{\mathbf{u}}(k))+\gamma(x)-\sum_{i=0}^{H_{p}-1} \ell\left(x_{s}, u_{s}\right) .
$$

Since $\sum_{i=0}^{H_{p}-1} \ell\left(x_{s}^{*}, u_{s}^{*}\right)$ and $\gamma(x)$ are independent of the decision variable $\tilde{\mathbf{u}}$ for a given initial state $x \in \mathbb{X}$, functions $\bar{L}(\tilde{\mathbf{x}}(k), \tilde{\mathbf{u}}(k))$ and $L(\tilde{\mathbf{x}}(k), \tilde{\mathbf{u}}(k))$ differ only by a constant. Therefore, the optimization problems in 16 and 22 have the same identical

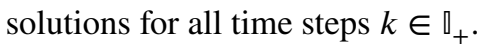

Lemma 3. The modified costs $\mathcal{L}$ and $\bar{V}_{f}$ satisfy the property

$$
\bar{V}_{f}\left(\left(A(\theta(k))+B(\theta(k)) K_{f}(\theta(k))\right) x(k)\right) \leq \bar{V}_{f}(x)-\mathcal{L}\left(x, K_{f}(\theta(k)) x\right)
$$

if and only if $V_{f}(k)$ and $\ell(k)$ satisfy Assumption 6

Proof. The proof follows from the results presented in ${ }^{30}$ by considering Remarks 2 and 3 and performing the proper manipulation of 21 ) by adding the term $\gamma(x)+\gamma\left(\left(A(\theta(k))+B(\theta(k)) K_{f}(\theta(k))\right) x(k)\right)$ to both sides.

Lemma 4. ${ }^{[32}$ Let $\rho(x): \mathcal{C} \rightarrow \mathbb{R}_{\geq 0}$ be a positive definite function that is defined on the compact $\operatorname{set} C$. Then, there exists a class $\mathcal{K}$ function $\zeta($.) such that

$$
\rho(x) \geq \zeta(|x|), \quad \forall x \in \mathcal{C} .
$$

Lemma 5. Let Assumptions 3 to 6 hold. The terminal cost $\bar{V}_{f}$ and rotated stage cost $\mathcal{L}$ satisfy the following inequalities:

$$
\begin{array}{lr}
\mathcal{L}(x, u) \geq \zeta\left(\left\|x-x_{s}^{*}\right\|\right) \geq 0, & \forall(x, u) \in \mathbb{Z} \\
\zeta\left(\left\|x-x_{s}^{*}\right\|\right) \leq \bar{V}_{f} \leq \hat{\zeta}\left(\left\|x-x_{s}^{*}\right\|\right), & \forall(x) \in \mathbb{X}_{f}
\end{array}
$$

where functions $\zeta($.) and $\hat{\zeta}($.) are of class $\mathcal{K}$.

Proof. From (19), 21a) and Assumption 3 it holds that $\mathcal{L}(x, u) \geq \rho\left(\left\|x-x_{s}^{*}\right\|\right)$ for all $(x, u) \in \mathbb{Z}$, which according to Lemma 4. leads to 27]. By following ${ }^{30}$, it can be shown from (25) and (27) that $\bar{V}_{f}(x) \geq \sum_{i=0}^{\infty} \mathcal{L}\left(x(i), K_{f}(\theta(k)) x(i)\right)$. Moreover, from Assumption $4 \bar{V}_{f}(x(k))$ is bounded and by $\bar{V}_{f}\left(x_{s}^{*}\right)=0$, thus, it can be upperbounded by a class $\mathcal{K}$ function, i.e., $\bar{V}_{f}(x) \leq$ $\hat{\zeta}\left(\left\|x-x_{s}^{*}\right\|\right)$ for all $x \in \mathbb{X}_{f}$. 
Theorem 1. Let Assumptions 3 to 6 hold. Then, the optimal state trajectory of (3) is asymptotically stable for all feasible initial states. The candidate Lyapunov function is $\bar{L}^{0}(x(k))$, and satisfies

$$
\begin{aligned}
\bar{L}^{0}(x(k)) & \leq \zeta\left(\left\|x-x_{s}^{*}\right\|\right), \\
\bar{L}^{0}(x(k+1))-\bar{L}^{0}(x(k)) & \leq-\underline{\zeta}\left(\left\|x-x_{s}^{*}\right\|\right),
\end{aligned}
$$

for all $x \in \mathbb{X}_{f}$, where $\underline{\zeta}($.$) being a class \mathcal{K}$ function.

Proof. The lower and upper bounds imposed by inequality (29) follow from Lemma 5 Condition (30) can be proved by considering the following optimal modified cost function:

$$
\left.\bar{L}(\tilde{\mathbf{x}}(k), \tilde{\mathbf{u}}(k))=\sum_{i=0}^{H_{p}-1} \mathcal{L}(x(i), u(i))+\bar{V}_{f}\left(x\left(H_{p}\right)\right)\right), \quad x \in \mathbb{X}_{f} .
$$

There is a feasible solution for the current state that gives optimal input and state sequences denoted as in [10i] and (10j]. A candidate input sequence and an associated state sequence for the next time step are chosen as follows:

$$
\begin{aligned}
& \overline{\mathbf{u}}(k+1)=\left\{u^{*}(1 \mid k), \ldots, u^{*}\left(H_{p} \mid k\right), K_{f}\left(x^{*}\left(H_{p} \mid k\right)\right)\right\}, \\
& \overline{\mathbf{x}}(k+1)=\left\{x^{*}(1 \mid k), \ldots, x^{*}\left(H_{p} \mid k\right), x^{*}\left(H_{p}+1 \mid k\right)\right\},
\end{aligned}
$$

where $x^{*}\left(H_{p}+1 \mid k\right)=\left(\left(A\left(\theta_{k}\right)+B\left(\theta_{k}\right) K_{f}\left(\theta_{k}\right)\right)\right) x^{*}\left(H_{p} \mid k\right)$. Due to the terminal constraint and Assumption 6 , it holds $x^{*}\left(H_{p}+\right.$ $1 \mid k) \in \mathbb{X}_{f}$. Furthermore, the cost is given by

$$
\begin{aligned}
\bar{L}(\overline{\mathbf{x}}(k+1), \overline{\mathbf{u}}(k+1)) & =\sum_{l=1}^{H_{p}-1} \mathcal{L}\left(x^{*}(i \mid k), u^{*}(i \mid k)\right)+\mathcal{L}\left(x^{*}\left(H_{p} \mid k\right), K_{f}\left(x^{*}\left(H_{p} \mid k\right)\right)\right)+\bar{V}_{f}\left(x^{*}\left(H_{p}+1 \mid k\right)\right) \\
& =\bar{L}^{0}(x(k))-\mathcal{L}\left(x(k), u^{*}(k \mid k)\right)+\mathcal{L}\left(x^{*}\left(H_{p} \mid k\right), K_{f}\left(x^{*}\left(H_{p} \mid k\right)\right)\right)-\bar{V}_{f}\left(x^{*}\left(H_{p} \mid k\right)\right)+\bar{V}_{f}\left(x^{*}\left(H_{p}+1 \mid k\right)\right) .
\end{aligned}
$$

From Assumption 6 and Lemma 3 it follows that

$$
\bar{L}(\overline{\mathbf{x}}(k+1), \overline{\mathbf{u}}(k+1)) \leq \bar{L}^{0}(x(k))-\mathcal{L}\left(x(k), u^{*}(0 \mid k)\right) .
$$

Since $\bar{L}^{0}(x(k+1)) \leq \bar{L}(\overline{\mathbf{x}}(k+1), \overline{\mathbf{u}}(k+1))$, hence, from (33) and Lemma 5 , it follows that

$$
\bar{L}^{0}(x(k+1))-\bar{L}^{0}(x(k)) \leq-\underline{\zeta}\left(\left\|x-x_{s}^{*}\right\|\right),
$$

which completes the proof.

\subsection{Computation of the terminal components}

For completely establishing the stability of the proposed EMPC approach, it is necessary to compute the terminal components that provide recursive feasibility.

Recursive feasibility is the property that for any initial condition $x(0 \mid k) \in \mathbb{X}$ solutions of $\left[16\right.$ fulfill $x(i \mid k) \in \mathbb{X}, i \in\left[1, H_{p}\right]$. Indeed, due to the presence of hard constraints in (16), feasible solutions may fail to exist. The property of recursive feasibility then ensures that, provided the system's state is updated according to its nominal dynamics, feasibility is preserved at all times. This fact is normally shown by induction. According to 27 , there are several ways of guaranteeing recursive feasibility, as e.g., using terminal equality constraints or using terminal sets. In this paper, the second option has been chosen.

By following $\frac{30}{}$, a systematic procedure is presented where a fixed terminal region around the optimal steady state was used. Then, terminal sets and quadratic cost functions are computed based on the LPV model (3).

Assumption 7. There exist a state-feedback controller $K(\theta(k))$, such that the closed-loop system

$$
\tilde{A}(\theta(k)):=A(\theta(k))+B(\theta(k)) K(\theta(k))
$$

is stable for all $\theta \in \Theta$.

The computation of the terminal components of (10) requires in addition a terminal control law, which is determined as follows:

$$
K_{f}(\theta(k))=K(\theta(k))\left(x-x_{s}^{*}\right)+u_{s}^{*},
$$


where $K(\theta(k))$ is the feedback gain satisfying Assumption 7, and the pair $\left(x_{s}^{*}, u_{s}^{*}\right)$ are elements of the optimal steady state and input trajectories. The terminal penalty is introduced based on the ellipsoidal level sets correlated to quadratic functions of the form

$$
V_{f}(x):=\frac{1}{2}\left(x-x_{s}^{*}\right)^{\top} P\left(x-x_{s}^{*}\right) .
$$

Therefore, the terminal regions are centered around the optimal state state pair $\left(x_{s}^{*}, u_{s}^{*}\right)$ and defined as

$$
\mathbb{X}_{f}:=\left\{x \in \mathbb{R} \mid\left(x-x_{s}^{*}\right)^{\top} P\left(x-x_{s}^{*}\right) \leq \delta\right\},
$$

where $\delta \in \mathbb{R}_{+}$. The scalar $\delta$ must ensure that the state and input constraints are perpetually satisfied under the use of terminal controller (35), i.e., $x(k) \in \mathbb{X}_{f} \subset \mathbb{X}$ and $K_{f}(\theta(k)) x(k) \in \mathbb{U}$ for all $k \in \mathbb{q}_{+}$. To derive an appropriate terminal function $V_{f}$ for the economic cost based on the LPV model in (3), the procedure in ${ }^{30}$ is suitably modified. First, assume that the economic costs $\ell\left(\right.$.) are twice continuously differentiable and let $\bar{\ell}(x(k)):=\ell\left(x(k), K_{f}(\theta(k)) x(k)\right)-\ell\left(x_{s}^{*}, u_{s}^{*}\right)$. Then, from ${ }^{30}$, for all $x \in \mathbb{X},\left(x_{s}^{*}, u_{s}^{*}\right) \in \mathbb{Z}$ and $k \in \mathbb{\mathbb { V }}_{\left[0, H_{p}-1\right]}$, there exists a matrix $Q$ such that $Q-\bar{\ell}(x(k)) \geq 0$. Moreover, the quadratic cost functional is defined as

$$
\ell_{q}(x):=\frac{1}{2}\left(x-x_{s}^{*}\right)^{\top} Q\left(x-x_{s}^{*}\right)+q^{\top}\left(x-x_{s}^{*}\right),
$$

where $q:=\bar{\ell}\left(x_{s}^{*}(k), u_{s}^{*}(k)\right)$ such that for all $x \in \mathbb{X}$ the inequality $\ell_{q}(x) \geq \bar{\ell}(x(k))+(1 / 2)\left(x-x_{s}^{*}\right)^{\top}\left(x-x_{s}^{*}\right)$ holds. Hence, the candidate terminal function is defined as

$$
V_{f}:=\sum_{i=0}^{\infty} \ell_{q}(x(i)),
$$

where $x(i+1)=A(\theta(i)) x(i)+B(i)\left(K(\theta(i))\left(x-x_{s}^{*}\right)+u_{s}^{*}\right)$ for all $i \in \mathbb{Q}_{+}$.

Let obtain an explicit definition of (39). From Assumption 7 and (35), the error dynamics are given by

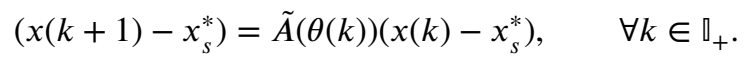

The so-called monodromy matrix of system (40) at time step $k$ is defined as

$$
\Psi_{k}:=\prod_{l=0}^{k} \tilde{A}(\theta(l)), \quad \forall k \in \mathbb{\square}_{+} .
$$

Then, according to (38), (40) and (41), the terminal function (39) can be written as follows:

$$
\begin{aligned}
V_{f} & =\frac{1}{2} \sum_{l=0}^{H_{p}-1}\left(x(l)-x_{s}^{*}\right)^{\top}\left(\sum_{i=0}^{\infty}\left(\Psi_{l}^{i}\right)^{\top} Q_{l}\left(\Psi_{l}^{i}\right)\right)\left(x(l)-x_{s}^{*}\right)+\bar{\ell}_{x}\left(x_{s}^{*}, u_{s}^{*}\right)^{\top} \sum_{i=0}^{\infty}\left(\Psi_{l}^{i}\right)\left(x(l)-x_{s}^{*}\right) \\
& =\frac{1}{2} \sum_{l=0}^{H_{p}-1}\left(x(l)-x^{*}\right)^{\top} P_{l}\left(x(l)-x_{s}^{*}\right)+q_{l}^{\top}\left(x(l)-x_{s}^{*}\right),
\end{aligned}
$$

with $x(l+1)=A(\theta(l)) x(l)+B(\theta(l))\left(K(\theta(l))\left(x(l)-x_{s}^{*}\right)+u_{s}^{*}\right)$ for all $l \in \mathbb{1}_{\left[0, H_{p}-1\right]}$ and $q_{l}^{\top}=\bar{\ell}\left(x_{s}^{*}, u_{s}^{*}\right)^{\top}\left(I-\Psi_{l}\right)^{-1}$. For the given $k$ and each $l \in \mathbb{1}_{\left[0, H_{p}-1\right]}$, matrices $P_{l}$ are the solutions to the discrete Lyapunov equations $\Psi_{l}^{\top} P_{l} \Psi_{l}-P_{l}=-Q_{l}$. Through (39), it follows that the candidate function $V_{f}$ satisfies the condition in Assumption 6. In fact, from Assumption 7 and suitable manipulation of [39]- $[42$, the following balance may be derived:

$$
\begin{aligned}
& V_{f}\left(\left(A(\theta(k))+B(\theta(k)) K_{f}(\theta(k))\right) x(k)\right)-V_{f}(x(k)) \\
& \quad=\frac{1}{2}\left(x(k)-x_{s}^{*}(k)\right)^{\top}\left(\tilde{A}(\theta(k))^{\top} P_{k} \tilde{A}(\theta(k))-P_{k}\right)\left(x(k)-x_{s}^{*}(k)\right)-\bar{\ell}_{x}\left(x_{s}^{*}, u_{s}^{*}\right)^{\top}\left(x(k)-x_{s}^{*}\right) .
\end{aligned}
$$

Hence, (39) and (43) yield the following Lyapunov equation:

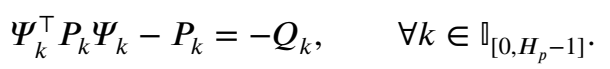

It is important to highlight that matrices $P_{k}$ and $K(\theta(k))$ must satisfy in addition the following condition:

$$
\tilde{A}(\theta(k))^{\top} P_{k+1} \tilde{A}(\theta(k))-P_{k} \leq 0, \quad \forall k \in \mathbb{\mathbb { 1 }}_{\left[0, H_{p}-1\right]} .
$$

to satisfy the positive invariant condition, i.e., if $x(k) \in \mathbb{X}_{f}$ then $\left(x(k), K_{f}(\theta(k)) x(k)\right) \in \mathbb{X}_{f}$ for all $k \in \mathbb{1}_{\geq 0}$.

The main problem to do so is that the matrices in (41) lead to nonlinear matrix inequalities that cannot be solved directly. Despite the mentioned difficulty for solving (44,, it is still conceivable to obtain a set of matrices satisfying Assumption 7 To do so, condition (44) should be relaxed and considering instead $\tilde{A}(\theta(k))^{\top} P_{k+1} \tilde{A}(\theta(k))-P_{k} \leq-Q_{k}$ for all $k \in \mathbb{1}_{\left[0, H_{p}-1\right]}$. The previous discussion is formalized by means of the result in Theorem 2 
Theorem 2. Consider the closed loop $x(k+1)=\left(A\left(\theta_{k}\right)+B\left(\theta_{k}\right) K_{f}\left(\theta_{k}\right)\right) x_{k}$ satisfying Assumption 4, control low (35) and the pair $\left(x_{s}^{*}, u_{s}^{*}\right) \in \mathbb{Z}$. Let $X_{k} \in \mathbb{S}_{++}^{n_{x}}, Y_{k} \in \mathbb{R}^{n_{u} \times n_{x}}$ and $\delta \in \mathbb{R}_{+}$be decision variables. By solving

$$
\max _{X_{k}>0, Y_{k} \in \mathbb{R}^{n_{u} x_{x}}, \delta \in \mathbb{R}_{+}}-\log \operatorname{det}\left(X_{0}\right)
$$

subject to

$$
\begin{aligned}
& {\left[\begin{array}{ccc}
X_{k} & \star & \star \\
A(\theta(k)) X_{k}+B(\theta(k)) Y_{k} & X_{k+1} & \star \\
Q^{1 / 2} X_{k} & 0_{n \times n} & \delta I_{n}
\end{array}\right] \geq 0,} \\
& {\left[\begin{array}{cc}
\left(h_{j}-\left(a_{j} x_{s}^{*}+b_{j} u_{s}^{*}\right)\right)^{2} & \star \\
X_{k} a_{j}^{\top}+Y_{k}^{\top} b_{j}^{\top} & X_{k}
\end{array}\right] \geq 0,}
\end{aligned}
$$

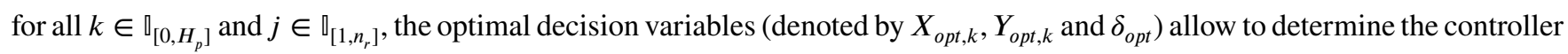

$$
K(\theta(k)):=Y_{k} X_{k}^{-1},
$$

and

$$
P_{k}:=X_{o p t, k}^{-1} \delta_{o p t} .
$$

$\forall k \in \mathbb{1}_{\left[0, H_{p}\right]} . P_{H_{p}}$ allows determining the terminal cost 36 and region 37 .

If problem (46) has a feasible solution, then Assumption 6 is satisfied.

Proof. First, it should be indicated that the solution of (46) presents the terminal sets defined in (37) for system $x(k+1)=$

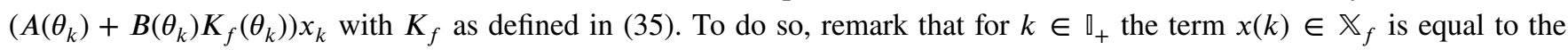
following quadratic functional condition:

$$
F_{0}=\left(x(k)-x_{s}^{*}\right)^{\top} P_{k}\left(x(k)-x_{s}^{*}\right)-\delta \leq 0 .
$$

Similarly, the requirement that $x(k+1) \in \mathbb{X}_{f}$ is equivalent to

$$
F_{1}=\left(x(k+1)-x_{s}^{*}\right)^{\top} P_{k+1}\left(x(k+1)-x_{s}^{*}\right)-\delta \leq 0 .
$$

According to ${ }^{33}$, the term that $x(k) \in \mathbb{X}_{f}$ implies $x(k+1) \in \mathbb{X}_{f}$, is equal to the existence of $\omega_{k}>0$, such that

$$
\left(x(k+1)-x_{s}^{*}\right)^{\top} P_{k+1}\left(x(k+1)-x_{s}^{*}\right)-\delta-\omega_{k}\left(\left(x(k)-x_{s}^{*}(k)\right)^{\top} P_{k}\left(x(k)-x_{s}^{*}-\delta\right) \leq 0 .\right.
$$

Therefore, following ${ }^{34}$, an equivalent linear matrix inequality condition can be established, i.e.,

$$
\left[\begin{array}{cc}
\tilde{A}(\theta(k))^{\top} P_{k+1} \tilde{A}(\theta(k))-\omega_{k} P_{k} & 0 \\
0 & \omega_{k}-\delta
\end{array}\right] \leq 0
$$

The above inequality can be decoupled in two inequalities: $0<\omega_{k} \leq \delta$, and

$$
\tilde{A}(\theta(k))^{\top} P_{k+1} \tilde{A}(\theta(k))-\omega_{k} P_{k} \leq 0 .
$$

As reported in ${ }^{35}$, there exists a $\omega_{k}$ such that $[50$ ) is equivalent to

$$
\tilde{A}(\theta(k))^{\top} P_{k+1} \tilde{A}(\theta(k))-P_{k} \leq-Q_{k} .
$$

By recovering $X_{k}$ and $Y_{k}$ according to 47] and applying the Schur complement to 46b, and pre- and post-multiplying the

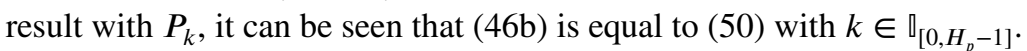

The terminal set satisfies state and input constraints for all $k \in \mathbb{1}_{\geq 0}$. This can be shown by retrieving $X_{k}$ and $Y_{k}$ according to (46) and applying the Schur complement to (46c), which gives

$$
\left(a_{j}+b_{j} K(\theta(k))\right)\left(\beta P_{k}^{-1}\right)\left(a_{j}+b_{j} K(\theta(k))^{\top} \leq\left(h_{j}-a_{j} \hat{x}_{s}^{*}-b_{j} \hat{u}_{s}^{*}\right)^{2},\right.
$$

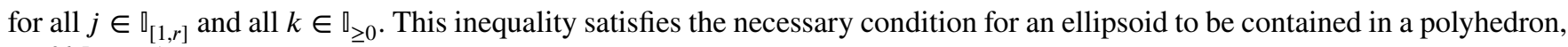
see ${ }^{36}$ Lemma 1 . Thus, (52) guarantees that, if $x(k) \in \mathbb{X}_{f}$ then $\left(x(k), K_{f}(\theta(k)) x(k)\right) \in \mathbb{X}_{f}$ for all $k \in \mathbb{1}_{\geq 0}$.

Finally, for proving (20) that guarantees the satisfaction of Assumption 6, (51) is pre- and post-multiplied by $\left(x(k)-x_{s}^{*}\right)^{\top}$ and $\left(x(k)-x_{s}^{*}\right)$ yielding the following inequality:

$$
\left(x(k)-x_{s}^{*}\right)^{\top}\left(\tilde{A}(\theta(k))^{\top} P_{k+1} \tilde{A}(\theta(k))-P_{k}\right)\left(x(k)-x_{s}^{*}\right) \leq-\left(x(k)-x_{s}^{*}\right)^{\top} Q_{k}\left(x(k)-x_{s}^{*}\right),
$$


for all $k \in \mathbb{q}_{+}$. By using (40, (41) and summing up (53) from $k=0$ to $k=H_{p}-1$, (53) can be rewritten as

$$
\left(x(k)-x_{s}^{*}\right)^{\top}\left(\Psi_{k}^{\top} P_{k+1} \Psi_{k}-P_{k}\right)\left(x(k)-x_{s}^{*}\right) \leq-\sum_{k=0}^{H_{p}-1}\left(x(k)-x_{s}^{*}(k)\right)^{\top} Q_{k}\left(x(k)-x_{s}^{*}\right) .
$$

Then, multiplying (53) by 0.5 and adding $-\bar{\ell}\left(x_{s}^{*}, u_{s}^{*}\right)^{\top}\left(x(k)-x_{s}^{*}\right)$ to both sides lead to

$$
\begin{aligned}
\frac{1}{2}\left(x(k)-x_{s}^{*}\right)^{\top}\left(\Psi_{k}^{\top} P_{k+1} \Psi_{k}-P_{k}\right)\left(x(k)-x_{s}^{*}\right)-\bar{\ell}\left(x_{s}^{*}(k), u_{s}^{*}\right)^{\top}\left(x(k)-x_{s}^{*}\right) \\
\leq-\frac{1}{2} \sum_{k=0}^{H_{p}-1}\left(x(k)-x_{s}^{*}\right)^{\top} Q_{k}\left(x(k)-x_{s}^{*}\right)-\bar{\ell}\left(x_{s}^{*}, u_{s}^{*}\right)^{\top}\left(x(k)-x_{s}^{*}\right) \\
\leq-\frac{1}{2}\left(x(k)-x_{s}^{*}(k)\right)^{\top} Q_{k}\left(x(k)-x_{s}^{*}(k)\right)-\bar{\ell}\left(x_{s}^{*}, u_{s}^{*}\right)^{\top}\left(x(k)-x_{s}^{*}\right) \\
\leq \bar{\ell}(x(k), u(k))=\ell\left(x(k), K_{f} x(k)\right)-\ell\left(x_{s}^{*}, u_{s}^{*}\right),
\end{aligned}
$$

for all $k \in \mathbb{q}_{\geq 0}$. The second inequality comes from the positive definiteness of $Q_{k}$, and the last inequality is obtained from the description of $\ell_{q}(x)$ in (38). Hence, according to (42) and (55), condition (20) is satisfied. Consequently, Theorem 2 is proved and the solution of (46) allows to obtain terminal components satisfying Assumption 6

Remark 6. Note that the controller in (47) is computed on-line at each iteration of the MPC controller by solving the LMI problem in Theorem 2] [R1-1] Such a controller is purely fictive and used only for calculating the terminal ingredients required to guarantee the EMPC stability. The scheduling variables $\theta(k)$ are treated in the same way as when solving the MPC LPV problem, i.e. by forecasting their value in the prediction horizon $H_{p}$ using (13). In this way, the complexity does not grow with the number of vaying parameters $\theta(k)$. Moreover, the number of LMIs in the prediction horizon $\boldsymbol{H}_{p}$ will kept at a reasonable size taking into account the usual rule of selecting the sampling time to have about 10 samples in the settling time of the system.

\section{5 | APPLICATION EXAMPLE}

\section{1 | Control-oriented model}

A case study based on a lab pasteurization process is considered. It is inspired in the small-scale plant PCT-23MKII, manufactured by Armfield (UK) ${ }^{37}$ (see Figure 2). The system represents an industrial High-Temperature Short-Time (HTST) process. In this process, the goal is to heat and preserve the product, which is typically a liquid, at a predetermined temperature during a minimum established period of time ${ }^{7}$. Figure 3 introduces a block diagram of the pasteurization simulation model, containing the feedback loops corresponding to the hot-water flow and power of the hat-water tank.

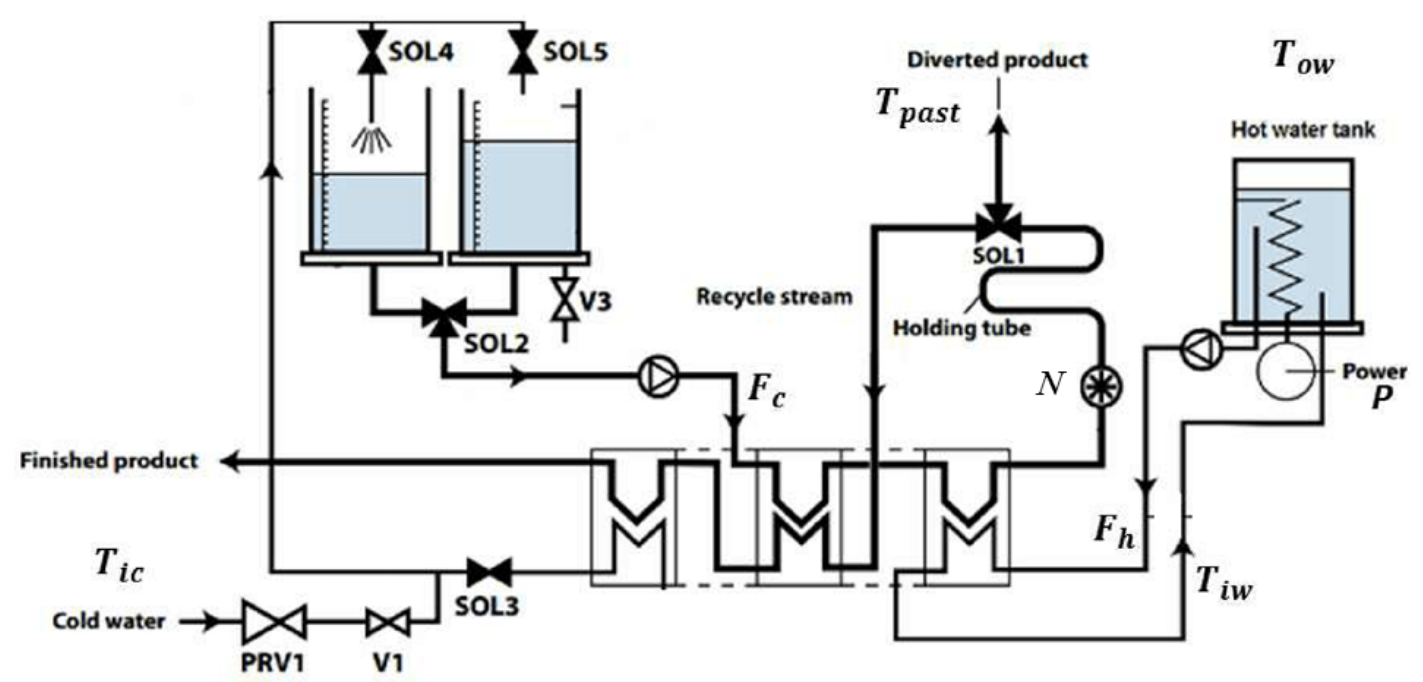

FIGURE 2 Pasteurization plant scheme. 
To model the whole pasteurization plant, dynamic models of such subsystems are obtained and represented in terms of behavioral equations. Then, dynamic equations are connected together towards stating the whole pasteurization model. Moreover, the mathematical models of the subsystems are obtained from the experimental data that is reported in 38 . Subsequently, models obtained as transfer functions are suitably stated by their equivalent controllable realizations in state space, with time-varying parameters $\theta(k)=\left[\begin{array}{ll}F_{h} & R\end{array}\right]$ where $F_{h}$ is the hot-water flow and the hot/cold-water flow ratio, $R$. Collecting all the information above, the discrete-time state-space LPV model can be written as in (3a), where

$$
\begin{aligned}
& A=\left[\begin{array}{ccccccc}
1+\frac{-T_{s}}{\tau_{1}\left(F_{h}(k)\right)} & 0 & 0 & 0 & 0 & 0 & 0 \\
0 & 1+\frac{-T_{s}}{\tau_{2}\left(F_{h}(k)\right)} & 0 & 0 & 0 & 0 & 0 \\
\frac{T_{s} K_{21}(R(k))}{\tau_{21}\left(F_{h}(k)\right)} & \frac{T_{s} K_{21}(R(k))}{\tau_{21}\left(F_{h}(k)\right)} & 1+\frac{-T_{s}}{\tau_{21}\left(F_{h}(k)\right)} & 0 & 0 & 0 & 0 \\
0 & 0 & 0 & 1+\frac{-T_{s}}{\tau_{12}\left(F_{h}(k)\right)} & 0 & \frac{T_{s} K_{12}(R(k))}{\tau_{12}\left(F_{h}(k)\right)} & 0 \\
0 & 0 & 0 & 0 & 1+\frac{-T_{s}}{\tau_{22}\left(F_{h}(k)\right)} & 0 & 0 \\
0 & 0 & 0 & 0 & 0 & 1+\frac{-T_{s}}{\tau_{f}} & 0 \\
0 & 0 & 0 & \frac{T_{s} K_{h t}}{\tau_{h t}} & \frac{T_{s} K_{h t}}{\tau_{h t}} & \frac{T_{s} K_{h t}}{\tau_{h t}} & 1+\frac{-T_{s}}{\tau_{h t}}
\end{array}\right],
\end{aligned}
$$

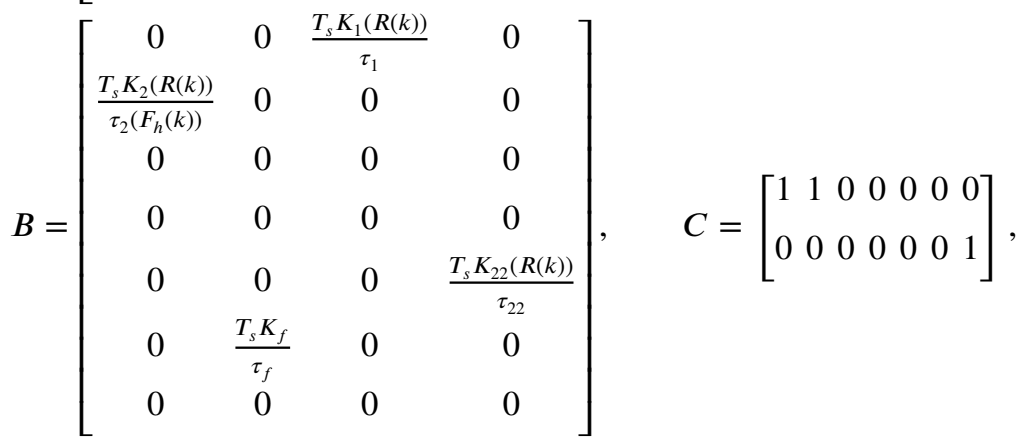

and, as can be seen in Figure 3. $x \in \mathbb{R}^{n_{x}}$ is the state vector composed among other of the hot-water flow, $F_{h}$, hot-water tank temperature, $T_{o w}$ and pasteurization temperature, $T_{p a s t} . u \in \mathbb{R}^{n_{u}}$ is the vector of manipulated variables that include among other the electrical power of the heater $P$ and the water pump speed $N . y \in \mathbb{R}^{n_{y}}$ is the vector of controlled variables that include of the hot-water tank temperature $T_{o w}$ and pasteurization temperatures $T_{p a s t}$. Moreover, the system matrices of the pasteurization plant model, including the time-varying parameters in function of the scheduling variables $\left(F_{h}\right.$ and $\left.R\right)$, can be expressed in the discrete-time state-space form as (3a), where $K$ are the static gains and the $\tau$ are the time constant of the transfer functions of the subsystems (see Figure 3).

\section{2 | Cost function parameters}

One of the key goals of a pasteurization process is to ensure that the pasteurization temperature is attained and preserved close to the set-point temperature for a pre-established time. However, the set-point is different for several products. Simultaneously, the decrease of energy consumption of the system considered an economic target should be achieved by minimizing the power of the resistor of the hot-water tank for reducing the cost of the heater. The controller minimizes a convex multiobjective stage cost function that includes economic aspects related to energy costs and to keeping the pasteurization temperature inside the safety bounds, while keeping smooth control input signals.

The EMPC objective function for the pasteurization process involves three operational goals: minimizing energy cost, maintaining safety bounds for temperature, and smoothing the control input signals.

\subsection{1 | Minimizing the energy costs}

One of the main control objective in the pasteurization process is to minimize the cost energy consumption of the system that includes minimizing the power of the hot-water tank for reducing the energy cost of the heater. Then, the cost function related to this objective can be formulated as

$$
\ell(k) \triangleq \alpha(k) W_{e} u(k) \Delta t,
$$




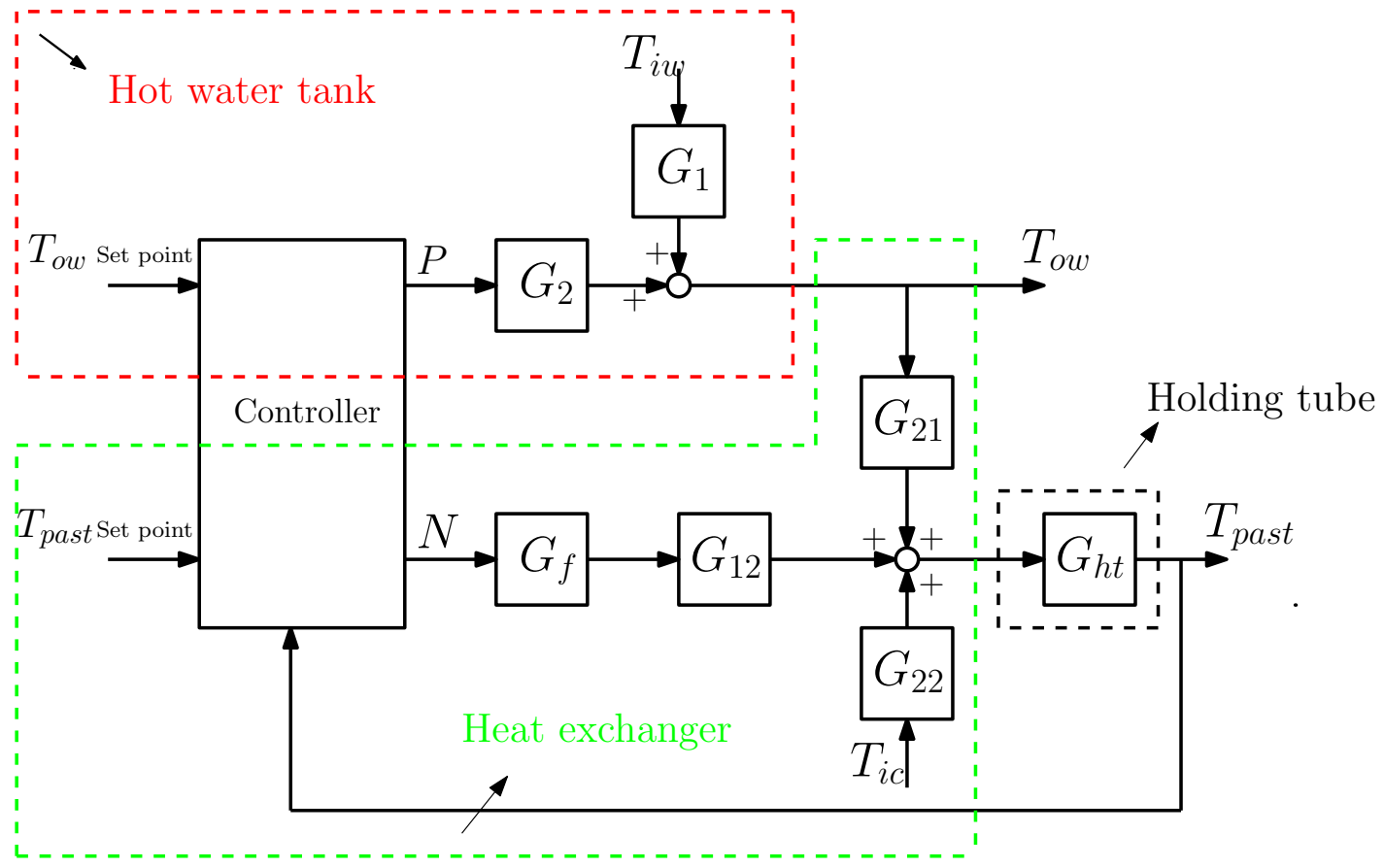

FIGURE 3 Control block diagram.

where $\alpha(k)$ is time-varying electricity cost that changes in each time instant $k$ according to the dynamic electricity tariff. Moreover, $W_{e}$ denotes the weighting term that indicates the prioritisation of the economic control objective and $\Delta t$ is the sampling time in seconds.

$$
\ell^{t}(k) \triangleq \xi(k)^{\top} W_{t} \xi(k),
$$

where $\xi>0$ is the considered slack variable added for preserving the feasibility of the optimization problem by stating the following soft constraint:

$$
C x(k+1) \geq y_{S}-\xi(k),
$$

being $W_{t}$ a diagonal positive definite matrix that determines the prioritisation of the safety objective.

\subsection{2 | Smoothing the control input signals}

Pumps and electric heater resistor are the actuators of the pasteurization plant. The control inputs determined by the EMPC controller should be smooth enough in order to extend the lifespan of the physical components. The cost function term for this control objective can be written as

$$
\ell^{\Delta}(k) \triangleq \Delta u(k)^{\top} W_{\Delta u} \Delta u(k),
$$

where the $\ell^{\Delta}(k)$ indicates the penalization of the slew rate $\Delta u(k) \triangleq u(k)-u(k-1)$ and $W_{\Delta u}$ is the weighting matrix of diagonal positive definite nature.

\subsection{3 | Multi-objective cost function}

According to the different control objectives already defined, the multi-objective cost function for the operational management of the pasteurization plant can be written as

$$
L_{k}=\left[\ell(k)+\ell^{\Delta}(k)\right] .
$$


TABLE 1 Comparison of control performance.

\begin{tabular}{lccc}
\hline Controller & $K P I_{e}$ & $K P I_{\Delta_{u}}$ & Simulation Time (in s) \\
\hline LPV-based EMPC & 1273 & 954 & 3354 \\
NEMPC & 1201 & 915 & 8717 \\
LPV-based TMPC & 2622 & 1243 & 3225 \\
\hline
\end{tabular}

\section{3 | Simulation results and discussion}

As mentioned before, one of the most important goals of the pasteurization process is to guarantee that the pasteurization temperature is reached and maintained as close as possible to the desired thermal temperature margin. At the same time, the reduction of energy consumption of the system expressed as an economic objective should be achieved by minimizing the power of the hot-water tank for reducing the cost consumption based on the power of the heater.

For this simulation, the input temperature of hot-water tank $T_{i w}$ and the temperature of cold-water $T_{i c}$ are maintained constant at $40^{\circ} \mathrm{C}$ and $30^{\circ} \mathrm{C}$, respectively. Moreover, the power of the electrical heater $P$ and water pump speed $N$ can take values in the range $[0,1.5] \mathrm{kW}$ and $[0,100] \mathrm{m}^{3} / \mathrm{s}$ as manipulated inputs, respectively. The states are constrained to be $[0,0,0,0,0,0,0]^{\top} \leq x(k) \leq[120,120,120,120,120,800,120]^{\top}$. The states of the LPV model shall be regulated from the initial state $x_{0}=[25,0,0,0,0,150,22]^{\top}$. The weighting matrices are $W_{e}=0.1, W_{t}=[80,0 ; 0,40], W_{\Delta u}=0.001$ and the prediction horizon has been selected as $H_{p}=6$.

According to (9a) and (60), the optimal steady-state values for the states of the pasteurization model (3) are obtained as $x_{s}^{*}=[28.87,48.14,17.29,13.485,98.58,56.83,70.02]^{\top}$ and $u_{s}^{*}=[264.58,17.06]^{\top}$. Moreover, as presented in Section 4. convergent behavior from the system to the optimal steady state $\left.\left(x_{s}^{*}, u_{s}^{*}\right)\right)$ can be ensured since the pasteurization system is strictly dissipative with respect to the supply rate $s(x, u):=\ell(x, u)-\ell\left(x_{s}^{*}, u_{s}^{*}\right)$, while the storage function is $\gamma=(1 / 2) x$. By solving (46) at each MPC iteration, it is obtained a sub-level $\delta=11.3697$ while matrices $P_{k}$, and the controller $K(\theta(k))$ can be found. Besides, according to matrices $P$, a terminal penalty as (36) can be defined, while $V_{f}$ in (20) can be satisfied for all $x \in \mathbb{X}_{f} \subset \mathbb{X}$ and $K_{f}=K_{k}\left(x-x_{s}^{*}\right)+u_{s}^{*}$.

To evaluate the advantage and economic efficiency of the proposed strategy based on the LPV model, a tracking MPC (TMPC) strategy is designed by using the LPV model of the pasteurization system. Such TMPC controller is designed through solving the following optimization problem:

$$
\min _{\mathbf{u}(k)} \sum_{l=0}^{H_{p}-1}\left\|x(l+1 \mid k)-x_{s}^{*}\right\|_{w_{1}}^{2}+\left\|u(l+1 \mid k)-u_{s}^{*}\right\|_{w_{2}}^{2}+\|\Delta u(l \mid k)\|_{w_{3}}^{2},
$$

subject to (3) and the same constraints considered by the EMPC controller. Moreover, the weighting matrices $w_{1} \in \mathbb{R}^{n_{x} \times n_{x}}$, $w_{2} \in \mathbb{R}^{n_{u} \times n_{u}}$ and $w_{3} \in \mathbb{R}^{n_{u} \times n_{u}}$ are determined to verify the priority of the several control objectives.

All simulations have been carried out on an i7 2.40-GHz Intel core processor with 12 GB of RAM running MATLAB R2016b, and the optimization problem is solved using YALMIP toolbox with CPLEX solver. In addition, the numeric assessment of the above-mentioned controllers has been carried through different key performance indicators (KPIs), which are detailed as follows:

$$
\begin{aligned}
K P I_{e} & =\frac{1}{n_{s}+1} \sum_{k=0}^{n_{s}} \alpha^{\top} u_{k} \Delta t, \\
K P I_{\Delta u} & =\frac{1}{n_{s}+1} \sum_{i=1}^{n_{u}} \sum_{k=0}^{n_{s}}\left(\Delta_{u}(i, k)\right)^{2},
\end{aligned}
$$

where $K P I_{e}$ denotes the average economic performance of the pasteurization process and $K P I_{\Delta u}$ evaluates the smoothness of the control inputs. Moreover, $n_{s} \in \mathbb{I}_{+}$is the number of seconds considered in the simulations. It should be noted that low values of $K P I_{e}$ and $K P I_{\Delta_{u}}$ imply better performance results. The comparison results of different KPIs as well as the simulation time between EMPC and TMPC strategies based on the LPV model are presented in Table 1. [R1-2, R1-3] Notice that the proposed LPV-based EMPC approach requires solving the LMI problem 46) and the QP problem (16) at each iteration. Thus, the computational time spent at each iteration includes the time required for solving both problems. 


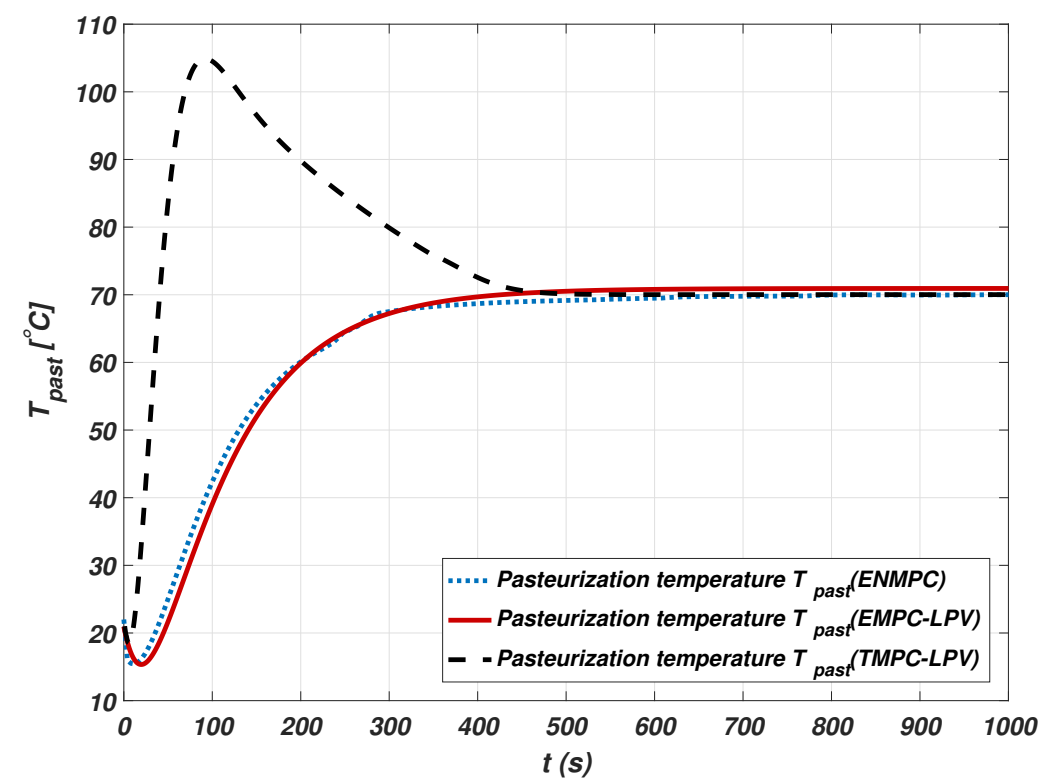

FIGURE 4 Evolution of controlled temperature of TMPC strategy and the EMPC based on the LPV model in (3)

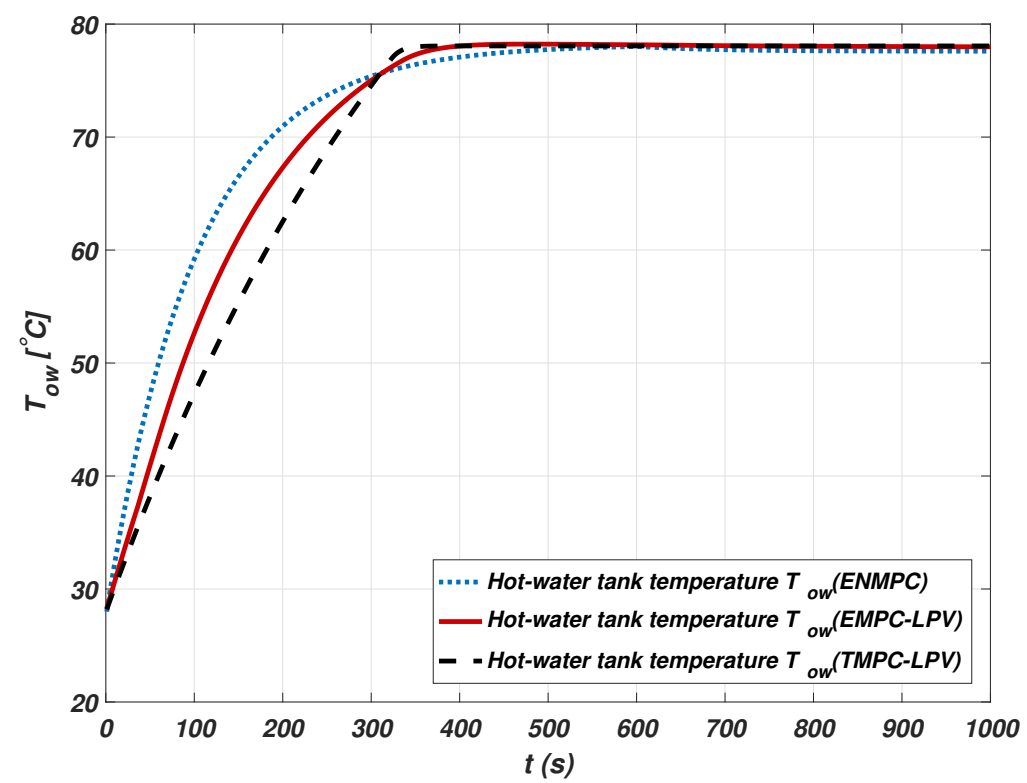

FIGURE 5 Evolution of controlled hot-water tank temperature of TMPC strategy and EMPC based on the LPV model in (3)

Figures 4 and 5 present the evolution of the controlled output temperature including $T_{p a s t}$ and $T_{o w}$. [R2-1] Notice that, in particular, the evolution of $T_{\text {past }}$ in Figure 4 shows a slight non-minimum phase behaviour. However, the effect of such a behaviour is here negligible. These output temperatures were obtained by using the proposed LPV-based EMPC strategy with hard constraints in order to save energy and avoid overheating the product. In Figure 4 it can be seen that $T_{p a s t}$ and $T_{o w}$ evolve within the expected ranges $T_{\text {past }} \in\left[65^{\circ} \mathrm{C}, 74^{\circ} \mathrm{C}\right]$ and $T_{\text {ow }} \in\left[76^{\circ} \mathrm{C}, 83^{\circ} \mathrm{C}\right]$, respectively. 

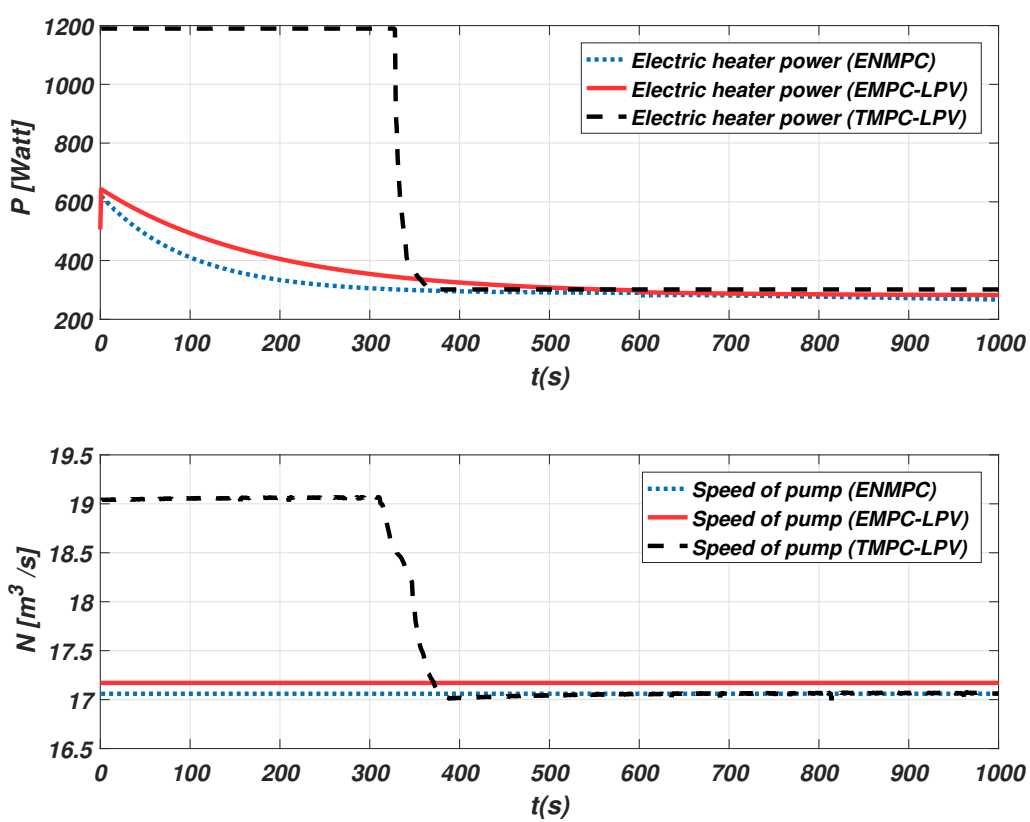

FIGURE 6 Evolution of control inputs of TMPC strategy and EMPC based on the LPV model in (3).

The TMPC is designed just for reaching the predefined the optimal steady state $\left(x_{s}^{*}, u_{s}^{*}\right)$. The results of the output temperature behaviour from the TMPC controller based on the LPV model designed to achieve the references are presented in Figure 4 From that figure, it can be seen that both $T_{p a s t}$ and $T_{o w}$ track the predetermined references. However, $T_{p a s t}$ shows overshoot in its transient.

Figure 6 shows the simulation results of the power of the electrical heater and speed of pump based for both EMPC and TMPC strategies. It can be seen that the optimal steady state values for $T_{\text {past }}$ and $T_{o w}$ are achieved by both controllers. However, by analyzing these results from the economic point of view, the EMPC controller requires less power of the electrical heater than the TMPC strategy, confirming the better economic performance of the first one. The economic cost in EMPC is almost reduced by three times compared to the TMPC strategy.

\section{6 | CONCLUSIONS}

This paper has proposed an Economic Model Predictive Control (EMPC) approach for Linear Parameter Varying (LPV) systems. An efficient implementation of the associated MPC optimization problem has been introduced based on transforming the LPV model into an linear time-varying one by using an estimation of the scheduling variables along the prediction horizon. This estimation is based on the optimal states/inputs determined from the solution of the previous optimization problem while running the receding horizon strategy. Using this approach, the solution of the proposed LPV-based EMPC scheme is possible by solving a series of Quadratic Programming (QP) problems at each time instant. This approach has allowed to reduce the computational burden compared to the solution of a nonlinear optimization problem that would result naturally from the LPV formulation. The stability of the proposed approach has been guaranteed by forcing the terminal state to vanish towards the optimal equilibrium point of the system (according to the desired control objectives). Moreover, the terminal constraint has been relaxed by using a constraint set around the terminal state instead of a single value and adding a penalty on the terminal state into the cost function. Besides, strict dissipativity has been established as a sufficient condition to prove stability. Finally, the effectiveness of the proposed LPV-based EMPC strategy has been shown by controlling a small-scale pasteurization system. The comparison assessment between the EMPC and the standard MPC approaches is performed. Results have shown the advantages of LPV-based EMPC in terms of economic cost minimization. As future research, the consideration of the mismatch between the predicted 
scheduling parameters based on the previously computed control sequence and the actual scheduling parameter will be addressed using a tube-based approach.

\section{References}

1. Hrovat D, Cairano SD, Tseng H, Kolmanovsky I. The development of model predictive control in automotive industry: A survey. In: Proceedings of the IEEE International Conference on Control Applications (CCA). IEEE. ; 2012; Dubrovnik, Croatia.

2. Qin S, Badgwell TA. A survey of industrial model predictive control technology. Control engineering practice 2003; 11(7): 733-764.

3. Raković S. Robust Model-Predictive Control: 1-11; London: Springer London . 2013.

4. Angeli D, Casavola A, Tedesco F. On average performance of economic model predictive control with time-varying cost and terminal constraints. In: American Control Conference (ACC). ACC. ; 2015; Chicago, USA.

5. Gros S, Zanon M, Quirynen R, Bemporad A, Diehl M. From linear to nonlinear MPC: bridging the gap via the real-time iteration. International Journal of Control 2020; 93(1): 62-80.

6. Diehl M, Bock HG, Schlöder JP. A Real-Time Iteration Scheme for Nonlinear Optimization in Optimal Feedback Control. SIAM Journal on Control and Optimization 2005; 43(5): 1714-1736.

7. Karimi Pour F, Puig Cayuela V, Ocampo-Martinez C. Health-aware model predictive control of pasteurization plant. In: Proceedings of the ACD'16-13th European Workshop on Advanced Control and Diagnosis. ADC. ; 2016; Lille, France.

8. Rugh WJ, Shamma JS. Research on gain scheduling. Automatica 2000; 36(10): 1401-1425.

9. Kwiatkowski A, Boll M, Werner H. Automated Generation and Assessment of Affine LPV Models. In: Proceedings of the 45th IEEE Conference on Decision and Control. IEEE. ; 2006; San Diego, USA.

10. Chang H, Krieger A, Astolfi A, Pistikopoulos E. Robust multi-parametric model predictive control for LPV systems with application to anaesthesia. Journal of Process Control 2014; 24(10): 1538-1547.

11. Rawlings JB, Mayne DQ. Model predictive control: Theory and design. Nob Hill Pub. . 2009.

12. Ellis M, Durand H, Christofides PD. A tutorial review of economic model predictive control methods. Journal of Process Control 2014; 24(8): 1156-1178.

13. Karimi Pour F, Puig V, Ocampo-Martinez C. Economic Predictive Control of a Pasteurization Plant using a Linear Parameter Varying Model. In: . 40. Elsevier. 2017 (pp. 1573-1578).

14. Ellis M, Christofides PD. Real-time economic model predictive control of nonlinear process systems. AIChE Journal 2015; 61(2): 555-571.

15. Diehl M, Amrit R, Rawlings JB. A Lyapunov function for economic optimizing model predictive control. IEEE Transactions on Automatic Control 2011; 56(3): 703-707.

16. Huang R, Harinath E, Biegler LT. Lyapunov stability of economically oriented NMPC for cyclic processes. Journal of Process Control 2011; 21(4): 501-509.

17. Heidarinejad M, Liu J, Christofides PD. Economic model predictive control of nonlinear process systems using Lyapunov techniques. AIChE Journal 2012; 58(3): 855-870.

18. Chen X, Heidarinejad M, Liu J, Christofides PD. Distributed economic MPC: Application to a nonlinear chemical process network. Journal of Process Control 2012; 22(4): 689-699. 
19. Faulwasser T, Grüne L, Müller MA. Economic Nonlinear Model Predictive Control. Foundations and Trends in Systems and Control 2018; 5(1): 1-98.

20. Grüne L, Semmler W, Stieler M. Using nonlinear model predictive control for dynamic decision problems in economics. Journal of Economic Dynamics and Control 2015; 60: 112 - 133.

21. Koehler J, Soloperto R, Muller MA, Allgower F. A computationally efficient robust model predictive control framework for uncertain nonlinear systems. IEEE Transactions on Automatic Control 2020.

22. Kunz K, Huck SM, Summers TH. Fast model predictive control of miniature helicopters. In: European Control Conference (ECC). EUCA. ; 2013: 1377-1382.

23. Cisneros PSG, Voss S, Werner H. Efficient Nonlinear Model Predictive Control via quasi-LPV representation. In: Proceedings of the 55th Conference on Decision and Control (CDC). IEEE. ; 2016; Las Vegas, USA.

24. González Cisneros PS, Werner H. Nonlinear model predictive control for models in quasi-linear parameter varying form. International Journal of Robust and Nonlinear Control 2020; 30(10): 3945-3959.

25. Boyd S, El Ghaoui L, Feron E, Balakrishnan V. Linear matrix inequalities in system and control theory. 15. Siam . 1994.

26. Mayne DQ. Model predictive control: Recent developments and future promise. Automatica 2014; 50(12): 2967-2986.

27. Angeli D, Müller MA. Economic Model Predictive Control: Some Design Tools and Analysis Techniques. In: Raković SV, Levine WS., eds. Handbook of Model Predictive ControlSpringer International Publishing. 2019 (pp. 145-167).

28. Cisneros P, Voss S, Werner H. Efficient Nonlinear Model Predictive Control via quasi-LPV representation. In: IEEE 55th Conference on Decision and Control (CDC). IEEE. ; 2016: 3216-3221.

29. Morato M, Normey-Rico J, Sename O. Novel qLPV MPC Design with Least-Squares Scheduling Prediction. IFACPapersOnLine 2019; 52(28): 158 - 163.

30. Amrit R, Rawlings JB, Angeli D. Economic optimization using model predictive control with a terminal cost. Annual Reviews in Control 2011; 35(2): 178-186.

31. Angeli D, Amrit R, Rawlings JB. On average performance and stability of economic model predictive control. IEEE transactions on automatic control 2012; 57(7): 1615-1626.

32. Kellett CM. A compendium of comparison function results. Mathematics of Control, Signals, and Systems 2014; 26(3): 339-374.

33. Boyd S, El-Ghaoui L, Feron E, Balakrishnan V, Yaz E. Linear matrix inequalities in system and control theory. Proceedings of the IEEE 1997; 85(4): 698-699.

34. Yu S, Böhm C, Chen H, Allgöwer F. Finite horizon model predictive control with ellipsoid mapping of uncertain linear systems. IET Control Theory \& Applications 2012; 6(18): 2820-2828.

35. Böhm C. Predictive control using semi-definite programming-efficient approaches for periodic systems and Lur'e systems. Logos-Verlag . 2011.

36. Böhm C, Yu S, Allgöwer F. Predictive control for constrained discrete-time periodic systems using a time-varying terminal region. In: Proceedings of the 14th International Conference on Methods and Models in Automation and Robotics. MMAR. ; 2009; Miedzyzdroje, Poland.

37. Armfield . Process Plant trainer PTC23-MKII, Instruction Manual. tech. rep., Armfield; UK: 2015.

38. Ibarrola JJ, Sandoval JM, García-Sanz M, Pinzolas M. Predictive control of a high temperature-short time pasteurisation process. Control Engineering Practice 2002; 10(7): 713-725. 